COMMUTING SMOOTHED PROJECTORS IN WEIGHTED NORMS WITH AN APPLICATION TO AXISYMMETRIC MAXWELL EQUATIONS

$$
\text { By }
$$

\title{
J. Gopalakrishnan
}

and

M. Oh

\section{IMA Preprint Series \# 2361}

( January 2011)

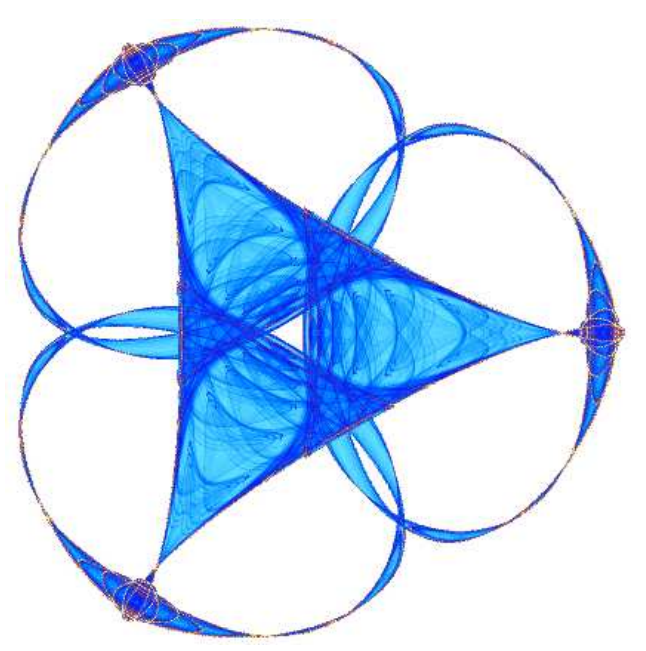

INSTITUTE FOR MATHEMATICS AND ITS APPLICATIONS

UNIVERSITY OF MINNESOTA 400 Lind Hall

207 Church Street S.E.

Minneapolis, Minnesota 55455-0436

Phone: 612-624-6066 Fax: 612-626-7370

URL: http://www.ima.umn.edu 


\title{
COMMUTING SMOOTHED PROJECTORS IN WEIGHTED NORMS WITH AN APPLICATION TO AXISYMMETRIC MAXWELL EQUATIONS
}

\author{
J. GOPALAKRISHNAN AND M. OH
}

\begin{abstract}
We construct finite element projectors that can be applied to functions with low regularity. These projectors are continuous in a weighted norm arising naturally when modeling devices with axial symmetry. They have important commuting diagram properties needed for finite element analysis. As an application, we use the projectors to prove a quasioptimality result for the edge finite element approximation of the axisymmetric (time-harmonic) Maxwell equations on bounded Lipschitz domains.
\end{abstract}

\section{INTRODUCTION}

Projectors (or interpolation operators) into finite element subspaces of Sobolev spaces are a fundamental ingredient in finite element error analyses. Every finite element has a canonical projector defined by its degrees of freedom. Often however, a technical problem arises, namely the unboundedness of the canonical projection in the Sobolev space where the solution is sought. To overcome this, many early analyses assumed that the solution is regular enough to be contained in the domain of the canonical projection. Clément [10] offered an alternative, at least for variational problems set in the Sobolev space $H^{1}$. The Clément interpolant is uniformly bounded in the $L^{2}$-norm and gives optimal approximation estimates. However, in the analysis of mixed methods, one needs projectors with further commutativity properties the Clément interpolant does not have. The importance of such commuting projectors has been evident early on [7, 22, 23] and has only been enhanced in more recent works [2]. The basic idea of Clément was generalized by Schöberl in $[25,26,27]$ to obtain similar projectors with the additional commutativity properties. His generalization was substantial, requiring several new ideas. In this paper, we will refer to operators obtained by his method as Schöberl projectors. Further refinements of Schöberl's ideas have been recently made in [8] (where the operators were called "smoothed projectors") and in [2] (where they were called "bounded cochain projectors"), but they do not extend to the case we intend to study here.

Our aim in this paper is to construct Schöberl projectors in the weighted norms arising in the study of axisymmetric Maxwell equations. Under axial symmetry, the time harmonic Maxwell equations in cylindrical coordinates $(r, \theta, z)$ decouple $[3,12]$ into two systems in the $r z$-halfplane. Due to the Jacobian arising from the change of variables however, we must work in weighted Sobolev spaces, where the (degenerate) weight function is the radial coordinate $r$. Let $L_{r}^{2}$ and $H_{r}$ (curl) denote the $r$-weighted analogues

This work was supported in part by the NSF under DMS-1014817. The authors also gratefully acknowledge the support from the IMA (Minneapolis) during their 2010-11 annual program. 
of the $L^{2}$ and $H$ (curl)-spaces (see their definitions in Section 2). To adapt the standard finite element techniques to these weighted spaces, we need commuting projectors in the weighted norms, in particular, the $H_{r}($ curl)-norm for treating axisymmetric electromagnetics. A commuting projector bounded in a more regular subspace of $H_{r}(\mathrm{curl})$ is already known [12]. A weighted Clément operator has been constructed in [4] for application to the axisymmetric Stokes problem. Even a commuting projector bounded in $H_{r}$ (curl) is also already known [11]. But, all these projectors are insufficient for various axisymmetric electromagnetic applications requiring low-regularity estimates, including the development of adaptive and multigrid algorithms. Hence we take up the task of constructing Schöberl projectors in $H_{r}$ (curl). In fact, anticipating other applications, we will do so for all the spaces in an exact sequence of weighted Sobolev spaces. As an example of how to apply the projector to obtain new results, we include a simple error analysis in weighted norms, under minimal regularity assumptions, for the axisymmetric indefinite time-harmonic Maxwell approximation following [21].

The outline of this paper follows the main steps in the construction of Schöberl projectors, as laid out in $[25,26,27]$.

(1) First, we recall existing nodal interpolation operators which are well defined for sufficiently regular functions in the weighted spaces (in Section 2) and summarize results of $[11,12,15]$ in this direction.

(2) Second, we introduce mesh dependent smoothing operators that are bounded in the weighted spaces (in Section 3) adapting the techniques in [27] to weighted spaces.

(3) Third, we compose the above two operations to form quasi-interpolation operators bounded in $L_{r}^{2}$ (in Section 4) as in [25, 27].

(4) The quasi-interpolation operators are not projectors. So in a final step, we compose with a finite dimensional inverse to obtain a projector, as in [26]. This construction is given in Section 5, where the main result (Theorem 5.1) appears.

Finally, in Section 6, as an application, we use the projectors to prove an error estimate for the finite element method applied to the axisymmetric Maxwell equations under minimal regularity assumptions.

\section{Preliminaries}

In this section, we recall the definitions of the weighted Sobolev spaces and the nodal interpolants of their corresponding finite element spaces.

Let $\mathbb{R}_{+}^{2}$ denote the $r z$-halfplane and $D \subseteq \mathbb{R}_{+}^{2}$ be a domain with Lipschitz boundary. Due to the axisymmetric applications we have in mind, we will further assume that the revolution of $D$ about the $z$-axis, namely $\Omega$, also has Lipschitz boundary. The weighted $L^{2}$-space is defined by

$$
L_{r}^{2}(D)=\left\{u: \int_{D}|u|^{2} r d r d z<\infty\right\} .
$$

This is a Hilbert space with the inner product $\left(u_{1}, u_{2}\right)_{r}=\int_{D} u_{1} u_{2} r d r d z$. For a general domain $G$ we denote the $L_{r}^{2}$-inner product on $G$ by $(\cdot, \cdot)_{r, G}$. Let $H_{r}^{1}(D)$ be the space of 
all functions in $L_{r}^{2}(D)$ whose first order distributional derivatives are also in $L_{r}^{2}(D)$. The norm and the seminorm on $H_{r}^{1}(D)$ are defined by

$$
\begin{aligned}
\|u\|_{H_{r}^{1}(D)} & =\left(\int_{D}\left(|u|^{2}+\left|\operatorname{grad}_{r z} u\right|^{2}\right) r d r d z\right)^{1 / 2}, \\
|u|_{H_{r}^{1}(D)} & =\left(\int_{D}\left|\operatorname{grad}_{r z} u\right|^{2} r d r d z\right)^{1 / 2},
\end{aligned}
$$

where $\operatorname{grad}_{r z} u=\left(\partial_{r} u, \partial_{z} u\right)$. Furthermore, for any real number $0<s<1, H_{r}^{s}(D)$ is defined as the Hilbert interpolation space $\left[H_{r}^{1}(D), L_{r}^{2}(D)\right]_{1-s}$ of index $1-s$ between the spaces $H_{r}^{1}(D)$ and $L_{r}^{2}(D)[6]$. In general, we will denote $\|\cdot\|_{X}$ and $|\cdot|_{X}$ to indicate the norm and the semi-norm respectively in a Sobolev space $X$.

Define the two-dimensional curl operator by

$$
\operatorname{curl}_{r z}\left(v_{r}, v_{z}\right)=\partial_{z} v_{r}-\partial_{r} v_{z},
$$

and set

$$
\boldsymbol{H}_{r}(\operatorname{curl} ; D)=\left\{\boldsymbol{v} \in L_{r}^{2}(D)^{2}: \operatorname{curl}_{r z} \boldsymbol{v} \in L_{r}^{2}(D)\right\} .
$$

$\boldsymbol{H}_{r}(\operatorname{curl} ; D)$ is a Hilbert space with the inner product

$$
\Lambda\left(\boldsymbol{v}_{1}, \boldsymbol{v}_{2}\right)=\left(\boldsymbol{v}_{1}, \boldsymbol{v}_{2}\right)_{r}+\left(\operatorname{curl}_{r z} \boldsymbol{v}_{1}, \operatorname{curl}_{r z} \boldsymbol{v}_{2}\right)_{r} .
$$

The induced norm is denoted by $\|\cdot\|_{\Lambda}$.

Now, let $\partial D=\Gamma_{0} \cup \Gamma_{1}$ where $\Gamma_{0}$ is the open segment forming the part of the boundary of $D$ on the axis of symmetry (z-axis), and $\Gamma_{1}=\partial D \backslash \Gamma_{0}$ denotes the remainder of the boundary (see Figure 2). Then, it is well-known that functions in $H_{r}^{1}(D)$ have traces in $L_{r}^{2}\left(\Gamma_{1}\right)$, i.e., for $u$ in $H_{r}^{1}(D)$, the trace $\left.u\right|_{\Gamma_{1}}$ makes sense as a function in $L_{r}^{2}\left(\Gamma_{1}\right)$, but trace on $\Gamma_{0}$ is not defined in general [18]. Additionally, since $\partial D$ is Lipschitz, the tangential trace operator on $\boldsymbol{H}_{r}(\operatorname{curl} ; D)$, which we will denote by $\left.\boldsymbol{v} \cdot \boldsymbol{t}\right|_{\Gamma_{1}}$, is proved in [12, Proposition 2.2] to be well-defined. Therefore, we can define the following closed subspaces of $H_{r}^{1}(D)$ and $\boldsymbol{H}_{r}(\operatorname{curl} ; D)$ :

$$
\begin{aligned}
H_{r, \triangleright}^{1}(D) & =\left\{u \in H_{r}^{1}(D):\left.u\right|_{\Gamma_{1}}=0\right\}, \\
\boldsymbol{H}_{r, \triangleright}(\operatorname{curl} ; D) & =\left\{\boldsymbol{v} \in \boldsymbol{H}_{r}(\operatorname{curl} ; D):\left.\boldsymbol{v} \cdot \boldsymbol{t}\right|_{\Gamma_{1}}=0\right\},
\end{aligned}
$$

where $\boldsymbol{t}$ denotes the unit tangent vector oriented counter-clockwise.

We are interested in constructing commuting projectors on $H_{r, \triangleright}^{1}(D), \boldsymbol{H}_{r, \triangleright}(\operatorname{curl} ; D)$, and $L_{r}^{2}(D)$ that require lower-order regularity. Assume that $D$ is meshed by a finite element triangulation $\mathscr{T}_{h}$ satisfying the usual geometrical conformity conditions [9]. We assume that $\mathscr{T}_{h}$ is quasiuniform with a representative mesh size $h$. The corresponding lowest order finite element subspace of $H_{r, \triangleright}^{1}(D)$ is the space of continuous functions that are linear on each mesh element, denoted by $V_{h, \triangleright}$. It has one nodal basis function $\phi_{\mathfrak{v}}$ for every mesh vertex $\mathfrak{v}$ not on $\Gamma_{1}$. The lowest order Nédélec subspace [22] of $\boldsymbol{H}_{r, \triangleright}(\operatorname{curl} ; D)$, on the same mesh is denoted by $\boldsymbol{W}_{h, \triangleright}$, with its corresponding edge basis functions $\left\{\phi_{\mathfrak{e}}\right\}$. And finally, let $S_{h}$ denote the $L_{r}^{2}(D)$-subspace with basis functions $\left\{\phi_{K}\right\}$ that are indicator functions of each mesh element $K$. That the sequence

$$
0 \longrightarrow V_{h, \triangleright} \stackrel{\operatorname{grad}_{r z}}{\longrightarrow} \boldsymbol{W}_{h, \triangleright} \stackrel{\operatorname{curl}_{r z}}{\longrightarrow} S_{h} \longrightarrow 0
$$


is exact is proved in [11, Appendix A] under the further assumptions that $\Gamma_{1}$ is connected and $D$ is simply connected. We also make the same assumptions throughout this paper.

We will need the following inequalities on polynomial spaces obtained by local homogeneity arguments. Let $K$ denote a triangle, $r(\boldsymbol{y})$ denote the value of the radial coordinate at a point $\boldsymbol{y} \in \mathbb{R}_{+}^{2}$,

$$
h_{K}=\operatorname{diam}(K), \quad r_{K}=\max _{\boldsymbol{x} \in K} r(\boldsymbol{x}),
$$

$\rho_{K}$ denote the diameter of the largest circle inscribed in $K$. Finally, let $P_{\ell}$ denote the space of polynomials of degree at most $\ell$ (for some $\ell \geqslant 0$ ). Throughout this paper, we use $C$ to denote a generic positive constant that is independent of $\left\{h_{K}\right\}$. Its value may differ at different occurrences and can depend on shape regularity ratio $h_{K} / \rho_{K}$, but not on $h_{K}$ by itself. A proof of the following proposition is indicated in Appendix A.

Proposition 2.1. For all $v \in P_{\ell}$,

$$
\begin{gathered}
\left\|\operatorname{grad}_{r z} v\right\|_{L_{r}^{2}(K)}^{2} \leqslant C h_{K}^{-2}\|v\|_{L_{r}^{2}(K)}^{2}, \quad\left\|\operatorname{grad}_{r z} v\right\|_{L^{\infty}(K)}^{2} \leqslant C h_{K}^{-2}\|v\|_{L^{\infty}(K)}^{2}, \\
r_{K} h_{K}^{2}\|v\|_{L^{\infty}(K)}^{2} \leqslant C\|v\|_{L_{r}^{2}(K)}^{2},
\end{gathered}
$$

on any $K \in \mathscr{T}_{h}$.

For smooth functions, the canonical interpolation operators of the finite element spaces $V_{h, \triangleright}, \boldsymbol{W}_{h, \triangleright}$, and $S_{h}$, namely, $I_{h}^{g}, I_{h}^{c}$, and $I_{h}^{o}$, resp., are such that the following diagram commutes:

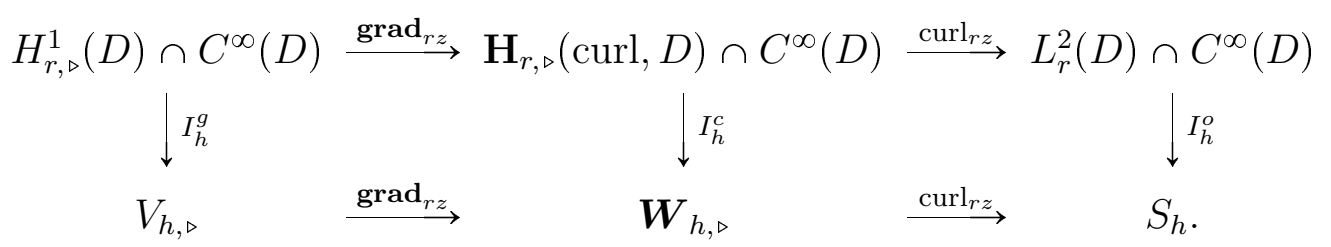

The projectors $I_{h}^{g}, I_{h}^{c}$, and $I_{h}^{o}$ are defined by the natural degrees of freedom (see $[15,12]$ for the modifications required in the degrees of freedom in weighted spaces), namely

$$
\begin{aligned}
& \left(I_{h}^{g} \omega\right)(\boldsymbol{x})=\sum_{\mathfrak{v} \in \mathscr{V}} \omega(\mathfrak{v}) \phi_{\mathfrak{v}}(\boldsymbol{x}), \\
& \left(I_{h}^{c} \boldsymbol{z}\right)(\boldsymbol{x})=\sum_{\mathfrak{e} \in \mathscr{E}}\left(\int_{\mathfrak{e}} \boldsymbol{z} \cdot \boldsymbol{t} d s\right) \phi_{\mathfrak{e}}(\boldsymbol{x}), \\
& \left(I_{h}^{o} s\right)(\boldsymbol{x})=\sum_{K \in \mathscr{T}_{h}}\left(\frac{1}{|K|} \int_{K} s d \boldsymbol{x}\right) \phi_{K},
\end{aligned}
$$

where $\mathscr{V}$ denotes the set of vertices in $\mathscr{T}_{h}$ not on $\Gamma_{1}, \mathscr{E}$ denotes the set of edges in $\mathscr{T}_{h}$ not on $\Gamma_{1},|K|$ denotes the measure of $K$, and $K \in \mathscr{T}_{h}$ denotes all triangles in $\mathscr{T}_{h}$. Note that $I_{h}^{g}$ and $I_{h}^{c}$ cannot be applied, however, to all functions in $H_{r, \triangleright}^{1}(D)$ and $\boldsymbol{H}_{r, \triangleright}(\operatorname{curl}, D)$, resp. They can at best be extended to more regular function spaces where the degrees of freedom make sense.

Our goal is to construct projectors, analogous to $I_{h}^{g}$ and $I_{h}^{c}$, satisfying the same commutativity properties in $(2.3)$, but bounded in $L_{r}^{2}(D)$ or $L_{r}^{2}(D)^{2}$. Therefore, in the next 

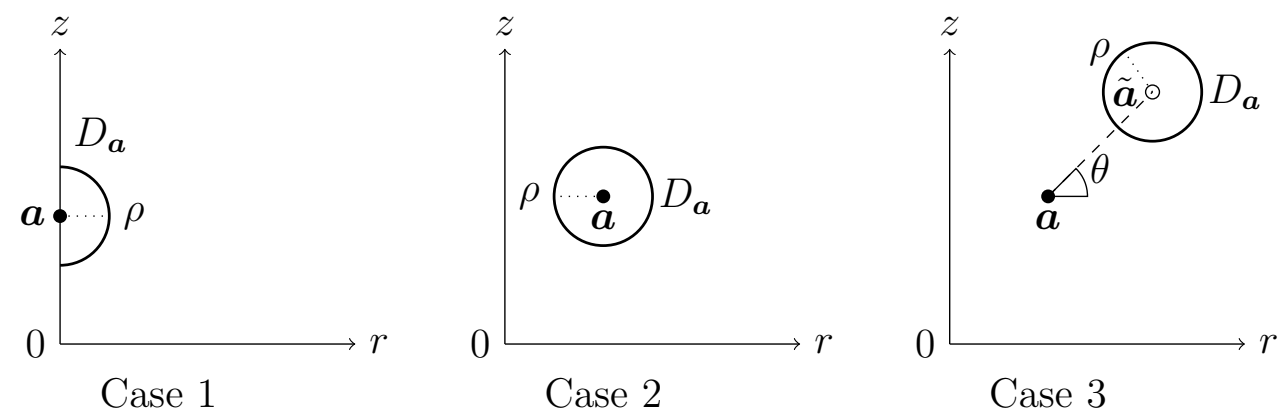

Figure 1. Domains $D_{\boldsymbol{a}}$ corresponding to point $\boldsymbol{a}$.

section, we define mesh dependent smoothers for functions in $L_{r}^{2}\left(\mathbb{R}_{+}^{2}\right)$ so that we can apply the classical nodal interpolation operators after we apply these smoothers to $L_{r}^{2}$-functions.

\section{Smoothing Operators}

The purpose of this section is to define smoothing operators $S^{g} u, S^{c} \boldsymbol{v}$ and $S^{o} w$, which we will use later. We introduce the notations and an intermediate result that will lead to Definition 3.1.

Let $\boldsymbol{a}=\left(a_{r}, a_{z}\right)$ be a point in $\mathbb{R}_{+}^{2}$ and let $D_{\boldsymbol{a}}$ be a closed disk of radius $\rho$, or its half, centered around $\boldsymbol{a}$ or $\tilde{\boldsymbol{a}}$, as shown in each of the three cases delineated in Figure 1. We need functions supported within these disks that will act as kernels within the integral smoothing operations to be defined. This will be obtained using the next proposition.

Proposition 3.1. Let $\ell \geqslant 0$. In all the three cases in Figure 1, there exists a function $\eta_{a}(r, z) \in P_{\ell}$ such that

$$
\begin{array}{ll}
\left(\eta_{\boldsymbol{a}}, p\right)_{r, D_{\boldsymbol{a}}}=p(a), & \forall p \in P_{\ell}, \\
\left\|\eta_{\boldsymbol{a}}\right\|_{L_{r}^{2}\left(D_{\boldsymbol{a}}\right)}^{2} \leqslant \frac{C}{\rho^{2} r_{\boldsymbol{a}}}, & \text { where } r_{\boldsymbol{a}}= \begin{cases}\rho, & \text { in Case 1, } \\
\min _{\boldsymbol{y} \in D_{\boldsymbol{a}}} r(\boldsymbol{y}), & \text { in Cases 2 and 3, }\end{cases} \\
\left\|\eta_{\boldsymbol{a}}\right\|_{L_{r}^{1}\left(D_{\boldsymbol{a}}\right)}^{2} \leqslant C \frac{r_{\boldsymbol{a}}+\rho}{r_{\boldsymbol{a}}}, & \text { where }\left\|\eta_{\boldsymbol{a}}\right\|_{L_{r}^{1}\left(D_{\boldsymbol{a}}\right)}=\int_{D_{\boldsymbol{a}}}\left|\eta_{a}\right| r(\boldsymbol{y}) d \boldsymbol{y} .
\end{array}
$$

A proof of Proposition 3.1 is included in Appendix A.

Next, let us define the "smoothing domains" $D_{\boldsymbol{a}}^{h}$ for each mesh vertex $\boldsymbol{a} \in \mathscr{T}_{h}$. Let $\delta>0$ be a global parameter. (We will need to choose it sufficiently small shortly.)

(1) If $\boldsymbol{a}$ is in (open) $\Gamma_{0}$, then $D_{\boldsymbol{a}}^{h}$ is set to the $D_{\boldsymbol{a}}$ in Case 1 of Figure 1 with $\rho=h \delta$.

(2) If $\boldsymbol{a}$ is in the interior of $D$, then $D_{\boldsymbol{a}}^{h}$ is set to $D_{\boldsymbol{a}}$ of Case 2 in Figure 1 with $\rho=h \delta$.

(3) If $\boldsymbol{a}$ is on $\Gamma_{1}$, then $D_{\boldsymbol{a}}^{h}$ is set to $D_{\boldsymbol{a}}$ of Case 3 in Figure 1 with $\rho=h \delta$ and $\tilde{\boldsymbol{a}}=\boldsymbol{a}+\operatorname{ch} \delta(\cos \theta, \sin \theta)$.

These smoothing domains are illustrated in Figure 2. We have to choose $\delta, c$, and $\theta$, noting that $\delta$ and $c$ are global constants, while $\theta \equiv \theta_{\boldsymbol{a}}$ can vary depending on $\boldsymbol{a}$. Let $D^{a}$ denote the "vertex patch" of $\boldsymbol{a}$, i.e., the domain formed by the union of all triangles in $\mathscr{T}_{h}$ connected to the mesh vertex $\boldsymbol{a}$ (see Figure 2). For $\boldsymbol{a}$ not on $\Gamma_{1}$, due to quasiuniformity, 


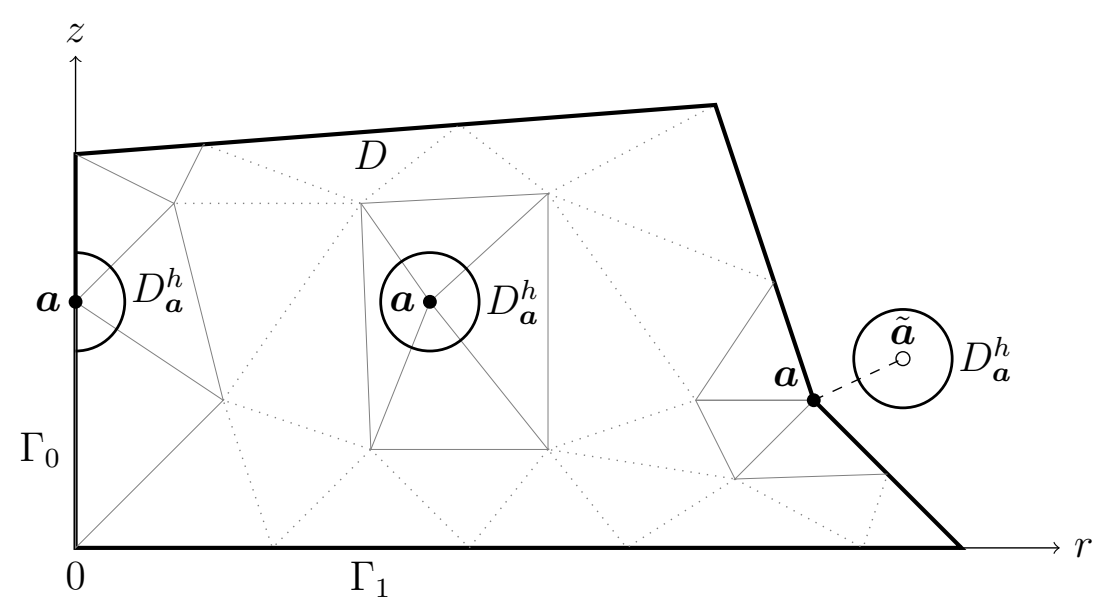

Figure 2. A domain, a few vertex patches, and associated smoothing domains

there exists $0<\delta_{0}<1$ such that $D_{\boldsymbol{a}}^{h} \subset D^{a}$ for all $\boldsymbol{a}$ and the smoothing domains of the distinct mesh vertices do not intersect. We choose the parameters such that the following holds: (i) For all $\boldsymbol{a}$ not on $\Gamma_{1}$, we have $D_{\boldsymbol{a}}^{h} \subset D^{a}$ and $r_{\boldsymbol{a}} \geqslant \delta h$. (This can be ensured by choosing a $\delta \leqslant \delta_{0}$ and making $\delta$ even smaller if necessary.) (ii) For all $\boldsymbol{a}$ on $\Gamma_{1}$, we have $D_{\boldsymbol{a}}^{h} \subset \mathbb{R}_{+}^{2} \backslash D$ and $r_{\boldsymbol{a}} \geqslant \delta h$. (This can be ensured by choosing $\theta$ and $c$ appropriately, due to the uniform cone property [17] implied by the Lipschitz regularity of the boundary.) Note that with these settings, we have

$$
r_{\boldsymbol{a}} \geqslant \delta h
$$

for all mesh vertices in $\mathscr{T}_{h}$, including vertices falling into Case 1.

We need some more notations to define the smoothing operators. We use [...] to denote the convex hull of its arguments. Accordingly, a triangle $K$ with vertices $\boldsymbol{a}_{1}, \boldsymbol{a}_{2}$, and $\boldsymbol{a}_{3}$ is $K=\left[\boldsymbol{a}_{1}, \boldsymbol{a}_{2}, \boldsymbol{a}_{3}\right]$. Its three edges are $\mathfrak{e}_{1}=\left[\boldsymbol{a}_{2}, \boldsymbol{a}_{3}\right], \mathfrak{e}_{2}=\left[\boldsymbol{a}_{3}, \boldsymbol{a}_{1}\right]$, and $\mathfrak{e}_{3}=\left[\boldsymbol{a}_{1}, \boldsymbol{a}_{2}\right]$. Let $D_{\boldsymbol{a}_{i}}^{h}$ be the smoothing domains introduced above for the vertices $\boldsymbol{a}_{i}(i=1,2,3)$, and let $\boldsymbol{y}_{i} \in D_{\boldsymbol{a}_{i}}^{h}$. Set

$$
\kappa_{i}\left(\boldsymbol{y}_{i}\right)=r\left(\boldsymbol{y}_{i}\right) \eta_{\boldsymbol{a}_{i}}\left(\boldsymbol{y}_{i}\right),
$$

where $\eta_{\boldsymbol{a}_{i}}$ is the function given by Proposition 3.1. We write $\kappa_{123}=\kappa_{1} \kappa_{2} \kappa_{3}$ and $\kappa_{12}=\kappa_{1} \kappa_{2}$, etc. For each $\boldsymbol{x} \in K$, let $\lambda_{i}(\boldsymbol{x})$ denote its barycentric coordinates in $K$ so that

$$
\boldsymbol{x}=\sum_{i=1}^{3} \lambda_{i}(\boldsymbol{x}) \boldsymbol{a}_{i}
$$

Following [26], we now define $\tilde{\boldsymbol{x}}_{\boldsymbol{y}}$ by

$$
\tilde{\boldsymbol{x}}_{\boldsymbol{y}}\left(\boldsymbol{x}, \boldsymbol{y}_{1}, \boldsymbol{y}_{2}, \boldsymbol{y}_{3}\right)=\sum_{i=1}^{3} \lambda_{i}(\boldsymbol{x}) \boldsymbol{y}_{i}
$$

and introduce these mesh dependent smoothers: 
Definition 3.1. For all $u, w \in L_{r}^{2}\left(\mathbb{R}_{+}^{2}\right)$ and $\boldsymbol{v} \in L_{r}^{2}\left(\mathbb{R}_{+}^{2}\right)^{2}$, define

$$
\begin{aligned}
& S^{g} u(\boldsymbol{x})=\int_{D_{\boldsymbol{a}_{1}}^{h}} \int_{D_{\boldsymbol{a}_{2}}^{h}} \int_{D_{a_{3}}^{h}} \kappa_{123} u\left(\tilde{\boldsymbol{x}}_{\boldsymbol{y}}\right) d \boldsymbol{y}_{3} d \boldsymbol{y}_{2} d \boldsymbol{y}_{1}, \\
& S^{c} \boldsymbol{v}(\boldsymbol{x})=\int_{D_{\boldsymbol{a}_{1}}^{h}} \int_{D_{\boldsymbol{a}_{2}}^{h}} \int_{D_{\boldsymbol{a}_{3}}^{h}} \kappa_{123}\left(\frac{\boldsymbol{d} \tilde{\boldsymbol{x}}_{\boldsymbol{y}}}{\boldsymbol{d} \boldsymbol{x}}\right)^{T} \boldsymbol{v}\left(\tilde{\boldsymbol{x}}_{\boldsymbol{y}}\right) d \boldsymbol{y}_{3} d \boldsymbol{y}_{2} d \boldsymbol{y}_{1}, \\
& S^{o} w(\boldsymbol{x})=\int_{D_{\boldsymbol{a}_{1}}^{h}} \int_{D_{\boldsymbol{a}_{2}}^{h}} \int_{D_{\boldsymbol{a}_{3}}^{h}} \kappa_{123} \operatorname{det}\left(\frac{\boldsymbol{d} \tilde{\boldsymbol{x}}_{\boldsymbol{y}}}{\boldsymbol{d} \boldsymbol{x}}\right) w\left(\tilde{\boldsymbol{x}}_{\boldsymbol{y}}\right) d \boldsymbol{y}_{3} d \boldsymbol{y}_{2} d \boldsymbol{y}_{1},
\end{aligned}
$$

for all $\boldsymbol{x} \in K$ and for each $K \in \mathscr{T}_{h}$.

It is easy to see that an equivalent way to write the last two operators is

$$
\begin{gathered}
S^{c} \boldsymbol{v}(\boldsymbol{x})=\int_{D_{\boldsymbol{a}_{1}}^{h}} \int_{D_{\boldsymbol{a}_{2}}^{h}} \int_{D_{\boldsymbol{a}_{3}}^{h}} \kappa_{123}\left(\sum_{i=1}^{3} \boldsymbol{y}_{i} \cdot \boldsymbol{v}\left(\tilde{\boldsymbol{x}}_{\boldsymbol{y}}\right) \operatorname{grad}_{r z} \lambda_{i}(\boldsymbol{x})\right) d \boldsymbol{y}_{3} d \boldsymbol{y}_{2} d \boldsymbol{y}_{1}, \\
S^{o} w(\boldsymbol{x})=\int_{D_{\boldsymbol{a}_{1}}^{h}} \int_{D_{\boldsymbol{a}_{2}}^{h}} \int_{D_{\boldsymbol{a}_{3}}^{h}} \kappa_{123}\left(\sum_{m=1}^{3}\left(\partial_{r} \lambda_{m}\right) \boldsymbol{y}_{m}\right) \times\left(\sum_{n=1}^{3}\left(\partial_{z} \lambda_{n}\right) \boldsymbol{y}_{n}\right) w\left(\tilde{\boldsymbol{x}}_{\boldsymbol{y}}\right) d \boldsymbol{y}_{3} d \boldsymbol{y}_{2} d \boldsymbol{y}_{1},
\end{gathered}
$$

where for two dimensional vectors $\boldsymbol{c}=\left(c_{r}, c_{z}\right)$ and $\boldsymbol{d}=\left(d_{r}, d_{z}\right)$, the (wedge) cross product yields the scalar $\boldsymbol{c} \times \boldsymbol{d}=c_{r} d_{z}-c_{z} d_{r}$. We will need to use the following properties of these smoothing operators.

Proposition 3.2. Let $u \in H^{1}\left(\mathbb{R}_{+}^{2}\right), \boldsymbol{v} \in \boldsymbol{H}_{r}\left(\operatorname{curl}, \mathbb{R}_{+}^{2}\right)$ and $w \in L_{r}^{2}\left(\mathbb{R}_{+}^{2}\right)$. Then the commutativity properties

$$
\begin{aligned}
S^{c}\left(\operatorname{grad}_{r z} u\right) & =\operatorname{grad}_{r z}\left(S^{g} u\right) \\
S^{o}\left(\operatorname{curl}_{r z} \boldsymbol{v}\right) & =\operatorname{curl}_{r z}\left(S^{c} \boldsymbol{v}\right)
\end{aligned}
$$

hold. Moreover, if $(i, j, k)$ is a permutation of $(1,2,3)$, the following identities hold:

$$
\begin{aligned}
S^{g} u\left(\boldsymbol{a}_{i}\right) & =\int_{D_{\boldsymbol{a}_{i}}^{h}} \kappa_{i} u\left(\boldsymbol{y}_{i}\right) d \boldsymbol{y}_{i}, \\
\int_{\mathfrak{e}_{k}} S^{c} \boldsymbol{v} \cdot \boldsymbol{t} d s & =\int_{D_{\boldsymbol{a}_{j}}^{h}} \int_{D_{\boldsymbol{a}_{k}}^{h}} \kappa_{i j} \int_{\left[\boldsymbol{y}_{i}, \boldsymbol{y}_{j}\right]} \boldsymbol{v} \cdot \boldsymbol{t} d s d \boldsymbol{y}_{i} d \boldsymbol{y}_{j}, \\
\int_{K} S^{o} w d \boldsymbol{x} & =\int_{D_{\boldsymbol{a}_{1}}^{h}} \int_{D_{\boldsymbol{a}_{2}}^{h}} \int_{D_{\boldsymbol{a}_{3}}^{h}} \kappa_{123} \int_{\left[\boldsymbol{y}_{1}, \boldsymbol{y}_{2}, \boldsymbol{y}_{3}\right]} w(\boldsymbol{z}) d \boldsymbol{z} d \boldsymbol{y}_{3} d \boldsymbol{y}_{2} d \boldsymbol{y}_{1} .
\end{aligned}
$$

Proof. The commutativity properties (3.9) and (3.10) hold by construction. It is easy to see this from Definition 3.1 and the Piola transformation.

To prove (3.11), we use (3.1) of Proposition 3.1, which implies that $\left(\eta_{\boldsymbol{a}_{2}}, 1\right)_{r, D_{\boldsymbol{a}_{2}}^{h}}=$ $\left(\eta_{\boldsymbol{a}_{3}}, 1\right)_{r, D_{\boldsymbol{a}_{3}}^{h}}=1$. Since $\tilde{\boldsymbol{x}}_{\boldsymbol{y}}=\boldsymbol{y}_{1}$ whenever $\boldsymbol{x}=\boldsymbol{a}_{1}$ (see (3.6)) we have

$$
S^{g} u\left(\boldsymbol{a}_{1}\right)=\int_{D_{\boldsymbol{a}_{1}}^{h}} \int_{D_{\boldsymbol{a}_{2}}^{h}} \int_{D_{\boldsymbol{a}_{3}}^{h}} \kappa_{123} u\left(\boldsymbol{y}_{1}\right) d \boldsymbol{y}_{3} d \boldsymbol{y}_{2} d \boldsymbol{y}_{1}=\left(\eta_{\boldsymbol{a}_{2}}, 1\right)_{r, D_{\boldsymbol{a}_{2}}^{h}}\left(\eta_{\boldsymbol{a}_{3}}, 1\right)_{r, D_{\boldsymbol{a}_{3}}^{h}} \int_{D_{\boldsymbol{a}_{1}}^{h}} \kappa_{1} u\left(\boldsymbol{y}_{1}\right) d \boldsymbol{y}_{1} .
$$

This proves (3.11) (since similar identities obviously hold for $i=2,3$ as well). 
To prove (3.12), let $\boldsymbol{q}(s)=(1-s) \boldsymbol{a}_{2}+s \boldsymbol{a}_{3}, 0 \leqslant s \leqslant 1$, and $\mathfrak{e}_{1}=\left[\boldsymbol{a}_{2}, \boldsymbol{a}_{3}\right]$. Then, we have that

$$
\begin{aligned}
\int_{\mathfrak{e}_{1}} & S^{c} \boldsymbol{v} \cdot \boldsymbol{t} d s=\int_{0}^{1} S^{c} \boldsymbol{v}(\boldsymbol{q}(s)) \boldsymbol{q}^{\prime}(s) d s \\
& =\int_{0}^{1} \int_{D_{\boldsymbol{a}_{1}}^{h}} \int_{D_{\boldsymbol{a}_{2}}^{h}} \int_{D_{\boldsymbol{a}_{3}}^{h}} \kappa_{123}\left(\sum_{i=1}^{3} \boldsymbol{y}_{i} \cdot \boldsymbol{v}\left((1-s) \boldsymbol{y}_{2}+s \boldsymbol{y}_{3}\right) \operatorname{grad}_{r z} \lambda_{i}\right) \cdot\left(\boldsymbol{a}_{3}-\boldsymbol{a}_{2}\right) d \boldsymbol{y}_{3} d \boldsymbol{y}_{2} d \boldsymbol{y}_{1} d s \\
& =\int_{0}^{1} \int_{D_{\boldsymbol{a}_{1}}^{h}} \int_{D_{\boldsymbol{a}_{2}}^{h}} \int_{D_{\boldsymbol{a}_{3}}^{h}} \kappa_{123}\left(\boldsymbol{y}_{3}-\boldsymbol{y}_{2}\right) \cdot \boldsymbol{v}\left((1-s) \boldsymbol{y}_{2}+s \boldsymbol{y}_{3}\right) d \boldsymbol{y}_{3} d \boldsymbol{y}_{2} d \boldsymbol{y}_{1} d s \\
& =\int_{D_{\boldsymbol{a}_{1}}^{h}} \int_{D_{\boldsymbol{a}_{2}}^{h}} \int_{D_{\boldsymbol{a}_{3}}^{h}} \kappa_{123} \int_{\left[\boldsymbol{y}_{2}, \boldsymbol{y}_{3}\right]} \boldsymbol{v} \cdot \boldsymbol{t} d s d \boldsymbol{y}_{3} d \boldsymbol{y}_{2} d \boldsymbol{y}_{1}, \\
& =\int_{D_{\boldsymbol{a}_{2}}^{h}} \int_{D_{\boldsymbol{a}_{3}}^{h}} \kappa_{23} \int_{\left[\boldsymbol{y}_{2}, \boldsymbol{y}_{3}\right]} \boldsymbol{v} \cdot \boldsymbol{t} d s d \boldsymbol{y}_{3} d \boldsymbol{y}_{2} .
\end{aligned}
$$

Here we have used the obvious identities $\operatorname{grad}_{r z} \lambda_{1} \cdot\left(\boldsymbol{a}_{3}-\boldsymbol{a}_{2}\right)=0, \operatorname{grad}_{r z} \lambda_{2} \cdot\left(\boldsymbol{a}_{3}-\boldsymbol{a}_{2}\right)=-1$, and $\operatorname{grad}_{r z} \lambda_{3} \cdot\left(\boldsymbol{a}_{3}-\boldsymbol{a}_{2}\right)=1$. The identities on the other edges follow similarly.

Finally, to prove (3.13), we use similar manipulations, with the additional observation that

$$
\frac{1}{\left(\sum_{m=1}^{3}\left(\partial_{r} \lambda_{m}(\boldsymbol{x})\right) \boldsymbol{y}_{m}\right) \times\left(\sum_{n=1}^{3}\left(\partial_{z} \lambda_{n}(\boldsymbol{x})\right) \boldsymbol{y}_{n}\right)}
$$

is the Jacobian arising from change of variables from $\boldsymbol{x}$ to $\tilde{\boldsymbol{x}}_{\boldsymbol{y}}$.

\section{QUASI-INTERPOLATION OPERATORS}

The next step is to study the quasi-interpolation operators that are compositions of the canonical interpolants and the smoothers of the previous section. From here on, let $u, w \in L_{r}^{2}(D)$ and $\boldsymbol{v} \in L_{r}^{2}(D)^{2}$. The trivial extension (by zero) of these functions to $L_{r}^{2}\left(\mathbb{R}_{+}^{2}\right)$ and $L_{r}^{2}\left(\mathbb{R}_{+}^{2}\right)^{2}$ will be denoted by $\tilde{u}, \tilde{w}$, and $\tilde{\boldsymbol{v}}$, resp.

Definition 4.1. Define the quasi-interpolation operators $R_{h}^{g}, R_{h}^{c}$, and $R_{h}^{o}$, by

$$
\begin{array}{rlrl}
R_{h}^{g} u & =I_{h}^{g} S^{g} \tilde{u}, & & \left(R_{h}^{g}: L_{r}^{2}(D) \longmapsto V_{h, \triangleright}\right), \\
R_{h}^{c} \boldsymbol{v}=I_{h}^{c} S^{c} \tilde{\boldsymbol{v}}, & & \left(R_{h}^{c}: L_{r}^{2}(D)^{2} \longmapsto \boldsymbol{W}_{h, \triangleright}\right), \\
R_{h}^{o} w=I_{h}^{o} S^{o} \tilde{w}, & & \left(R_{h}^{o}: L_{r}^{2}(D)^{2} \longmapsto S_{h}\right) .
\end{array}
$$

Note that these operators are not projectors as they do not preserve functions that are already in the finite element spaces. In the remainder of this section, we will establish two categories of properties of these operators. The first consist of commutativity identities, collected in Lemma 4.1. The second category, collected in Lemma 4.2, consists of norm estimates. In particular, we will prove that these quasi-interpolation operators are uniformly bounded in the weighted $L_{r}^{2}$-norm. 
Lemma 4.1. $R_{h}^{g}, R_{h}^{c}$, and $R_{h}^{o}$ satisfy the following commutativity properties:

$$
\begin{aligned}
\operatorname{grad}_{r z}\left(R_{h}^{g} u\right) & =R_{h}^{c}\left(\operatorname{grad}_{r z} u\right), & & \forall u \in H_{r, \triangleright}^{1}(D), \\
\operatorname{curl}_{r z}\left(R_{h}^{c} \boldsymbol{v}\right) & =R_{h}^{o}\left(\operatorname{curl}_{r z} \boldsymbol{v}\right), & & \forall \boldsymbol{v} \in \boldsymbol{H}_{r, \triangleright}(\operatorname{curl}, D) .
\end{aligned}
$$

Proof. This is obvious from the commutativity properties in Proposition 3.2 and (2.3).

Lemma 4.2. There exists a constant $C$ independent of $h$ and $\delta$ such that

$$
\begin{array}{rlrl}
\left\|R_{h}^{g} u\right\|_{L_{r}^{2}(D)}^{2} \leqslant \frac{C}{\delta^{3}}\|u\|_{L_{r}^{2}(D)}^{2}, & & \forall u \in L_{r}^{2}(D), \\
\left\|R_{h}^{g} u_{h}-u_{h}\right\|_{L_{r}^{2}(D)}^{2} \leqslant C \delta^{2}\left\|u_{h}\right\|_{L_{r}^{2}(D)}^{2}, & & \forall u_{h} \in V_{h, \triangleright}, \\
\left\|R_{h}^{c} \boldsymbol{v}\right\|_{L_{r}^{2}(D)}^{2} \leqslant \frac{C}{\delta^{3}}\|\boldsymbol{v}\|_{L_{r}^{2}(D)}^{2}, & & \forall \boldsymbol{v} \in L_{r}^{2}(D)^{2}, \\
\left\|R_{h}^{c} \boldsymbol{v}_{h}-\boldsymbol{v}_{h}\right\|_{L_{r}^{2}(D)}^{2} \leqslant C \delta^{2}\left\|\boldsymbol{v}_{h}\right\|_{L_{r}^{2}(D)}^{2}, & \forall \boldsymbol{v}_{h} \in \boldsymbol{W}_{h, \triangleright}, \\
\left\|R_{h}^{o} w\right\|_{L_{r}^{2}(D)}^{2} \leqslant \frac{C}{\delta}\|w\|_{L_{r}^{2}(D)}^{2}, & \forall w \in L_{r}^{2}(D), \\
\left\|R_{h}^{o} w_{h}-w_{h}\right\|_{L_{r}^{2}(D)}^{2} \leqslant C \delta^{2}\left\|w_{h}\right\|_{L_{r}^{2}(D)}^{2}, & \forall w_{h} \in S_{h} .
\end{array}
$$

Proof. Let $K=\left[\boldsymbol{a}_{1}, \boldsymbol{a}_{2}, \boldsymbol{a}_{3}\right]$ be a fixed triangle in $\mathscr{T}_{h}$, and let $D_{K}$ denote the union of $D_{\boldsymbol{a}_{1}}^{h}$, $D_{\boldsymbol{a}_{2}}^{h}$, and $D_{\boldsymbol{a}_{3}}^{h}$.

Proof of (4.3). Due to the shape regularity of $\mathscr{T}_{h}$ and the fact that $\tilde{u}$ is the extension of $u$ by zero, it suffices to prove local estimate

$$
\left\|R_{h}^{g} u\right\|_{L_{r}^{2}(K)}^{2} \leqslant \frac{C}{\delta^{3}}\|\tilde{u}\|_{L_{r}^{2}\left(D_{K}\right)}^{2}
$$

The nodal values of $R_{h}^{g} u$ on $K$ equal $S^{g} \tilde{u}\left(\boldsymbol{a}_{i}\right)$, which can be estimated by

$$
\begin{aligned}
\left|S^{g} \tilde{u}\left(\boldsymbol{a}_{i}\right)\right| & =\left|\int_{D_{\boldsymbol{a}_{i}}^{h}} \kappa_{i} \tilde{u}\left(\boldsymbol{y}_{i}\right) d \boldsymbol{y}_{i}\right| & & \text { by }(3.11), \\
& =\left|\left(\eta_{\boldsymbol{a}_{i}}, \tilde{u}\right)_{r, D_{\boldsymbol{a}_{i}}^{h}}\right| & & \text { by }(3.5), \\
& \leqslant\left\|\eta_{\boldsymbol{a}_{i}}\right\|_{L_{r}^{2}\left(D_{\boldsymbol{a}_{i}}^{h}\right)}\|\tilde{u}\|_{L_{r}^{2}\left(D_{\boldsymbol{a}_{i}}^{h}\right)}, & & \\
& \leqslant \frac{C}{\sqrt{(h \delta)^{2} r_{\boldsymbol{a}_{i}}}}\|\tilde{u}\|_{L_{r}^{2}\left(D_{\boldsymbol{a}_{i}}^{h}\right)} & & \text { by Proposition 3.1, (3.2). }
\end{aligned}
$$

Therefore,

$$
\begin{aligned}
\left\|R_{h}^{g} u\right\|_{L_{r}^{2}(K)}^{2} & =\int_{K}\left|\sum_{i=1}^{3} S^{g} \tilde{u}\left(\boldsymbol{a}_{i}\right) \lambda_{i}(\boldsymbol{x})\right|^{2} r(\boldsymbol{x}) d \boldsymbol{x} \\
& \leqslant C \sum_{i=1}^{3}\left|S^{g} \tilde{u}\left(\boldsymbol{a}_{i}\right)\right|^{2} \int_{K}\left|\lambda_{i}(\boldsymbol{x})\right|^{2} r(\boldsymbol{x}) d \boldsymbol{x} \\
& \leqslant C \sum_{i=1}^{3} \frac{1}{(h \delta)^{2} r_{\boldsymbol{a}_{i}}}\|\tilde{u}\|_{L_{r}^{2}\left(D_{\boldsymbol{a}_{i}}^{h}\right)}^{2} h^{2} r_{K}
\end{aligned}
$$


Since $r_{K} \leqslant r_{\boldsymbol{a}_{i}}+C h$, the ratio $r_{K} / r_{\boldsymbol{a}_{i}}$ can be bounded by

$$
\frac{r_{K}}{r_{a_{i}}} \leqslant 1+\frac{C h}{r_{a_{i}}} \leqslant 1+\frac{C}{\delta}
$$

where we have used (3.4). This proves (4.3).

Proof of (4.4). Again, we only need to prove the local estimate

$$
\left\|R_{h}^{g} u_{h}-u_{h}\right\|_{L_{r}^{2}(K)} \leqslant C \delta\left\|\tilde{u}_{h}\right\|_{L_{r}^{2}\left(D_{K}\right)}
$$

because $\tilde{u}_{h}$ is the extension of $u_{h}$ by zero and $\mathscr{T}_{h}$ is quasiuniform. First observe that

$$
\begin{aligned}
& \left|\left(S^{g} \tilde{u}_{h}\right)\left(\boldsymbol{a}_{i}\right)-u_{h}\left(\boldsymbol{a}_{i}\right)\right|=\int_{D_{\boldsymbol{a}_{i}}^{h}} \kappa_{i}\left(\tilde{u}_{h}\left(\boldsymbol{y}_{i}\right)-u_{h}\left(\boldsymbol{a}_{i}\right)\right) d \boldsymbol{y}_{i}, \\
& \quad \leqslant \max _{\boldsymbol{y}_{i} \in D_{\boldsymbol{a}_{i}}^{h}}\left|\boldsymbol{a}_{i}-\boldsymbol{y}_{i}\right|\left\|\operatorname{grad}_{r z} \tilde{u}_{h}\right\|_{L^{\infty}\left(D_{\boldsymbol{a}_{i}}^{h}\right)}\left\|\eta_{\boldsymbol{a}_{i}}\right\|_{L_{r}^{1}\left(D_{\boldsymbol{a}_{i}}^{h}\right)} \\
& \quad \leqslant C h \delta\left\|\operatorname{grad}_{r z} \tilde{u}_{h}\right\|_{L^{\infty}\left(D_{\boldsymbol{a}_{i}}^{h}\right)} \frac{r_{\boldsymbol{a}_{i}}+h \delta}{r_{\boldsymbol{a}_{i}}} \\
& \quad \leqslant C h \delta\left\|\operatorname{grad}_{r z} \tilde{u}_{h}\right\|_{L^{\infty}\left(D_{\boldsymbol{a}_{i}}^{h}\right)}
\end{aligned}
$$

Hence,

$$
\begin{aligned}
\left\|R_{h}^{g} u_{h}-u_{h}\right\|_{L_{r}^{2}(K)}^{2} & \leqslant C \sum_{i=1}^{3}\left|\left(S^{g} \tilde{u}_{h}\right)\left(\boldsymbol{a}_{i}\right)-u_{h}\left(\boldsymbol{a}_{i}\right)\right|^{2} \int_{K}\left|\lambda_{i}(\boldsymbol{x})\right|^{2} r(\boldsymbol{x}) d \boldsymbol{x}, \\
& \leqslant C \sum_{i=1}^{3}(h \delta)^{2}\left\|\operatorname{grad}_{r z} \tilde{u}_{h}\right\|_{L^{\infty}\left(D_{\boldsymbol{a}_{i}}^{h}\right)}^{2} h^{2} r_{K} \\
& \leqslant C(h \delta)^{2}\left\|\operatorname{grad}_{r z} \tilde{u}_{h}\right\|_{L_{r}^{2}\left(D_{K}\right)}^{2} \leqslant C \delta^{2}\left\|\tilde{u}_{h}\right\|_{L_{r}^{2}\left(D_{K}\right)}^{2}
\end{aligned}
$$

where we have used Proposition 2.1 in the last step. This proves (4.10).

Proof of (4.5). Let $C_{K}$ denote the convex hull of $D_{\boldsymbol{a}_{1}}^{h}, D_{\boldsymbol{a}_{2}}^{h}$, and $D_{\boldsymbol{a}_{3}}^{h}$. For the same reason as in the previous proofs, it suffices to prove the local estimate

$$
\left\|R_{h}^{c} \boldsymbol{v}\right\|_{L_{r}^{2}(K)}^{2} \leqslant \frac{C}{\delta^{3}}\|\tilde{\boldsymbol{v}}\|_{L_{r}^{2}\left(C_{K}\right)}^{2} .
$$

Let $\mathfrak{e}_{i}$ denote the edge connecting $\boldsymbol{a}_{j}$ and $\boldsymbol{a}_{k}$. Recall that throughout, $(i, j, k)$ denotes a permutation of $(1,2,3)$. Since $\left.R_{h}^{c} \boldsymbol{v}\right|_{K}=\sum_{i=1}^{3}\left(\int_{\mathfrak{e}_{i}} S^{c} \tilde{\boldsymbol{v}} \cdot \boldsymbol{t} d s\right) \phi_{\mathfrak{e}_{i}}$, with $\phi_{\mathfrak{k}_{i}}= \pm\left(\lambda_{j} \operatorname{grad}_{r z} \lambda_{k}-\right.$ $\left.\lambda_{k} \operatorname{grad}_{r z} \lambda_{j}\right)$, and since $\left\|\phi_{\mathfrak{e}_{i}}\right\|_{L_{r}^{2}(K)}^{2} \leqslant C r_{K}$, we have

$$
\begin{aligned}
\left\|R_{h}^{c} \boldsymbol{v}\right\|_{L_{r}^{2}(K)}^{2} & =\int_{K}\left|\sum_{i=1}^{3} \phi_{\mathfrak{e}_{i}}(\boldsymbol{x}) \int_{\mathfrak{e}_{i}} S^{c} \tilde{\boldsymbol{v}} \cdot \boldsymbol{t} d s\right|^{2} r(\boldsymbol{x}) d \boldsymbol{x}, \\
& \leqslant C \sum_{i=1}^{3}\left|\int_{\mathfrak{e}_{i}} S^{c} \tilde{\boldsymbol{v}} \cdot \boldsymbol{t} d s\right|^{2}\left\|\phi_{\mathfrak{e}_{i}}\right\|_{L_{r}^{2}(K)}^{2} \leqslant C r_{K} \sum_{i=1}^{3}\left|\int_{\mathfrak{e}_{i}} S^{c} \tilde{\boldsymbol{v}} \cdot \boldsymbol{t} d s\right|^{2} .
\end{aligned}
$$




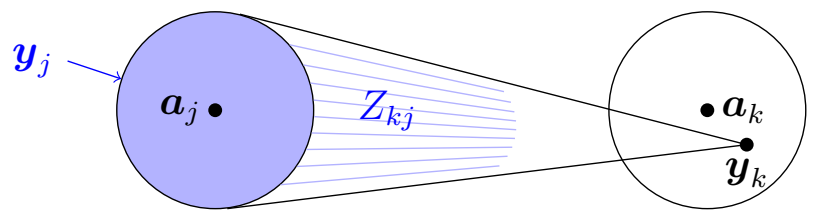

FiguRE 3. Illustration of the change of variable in the proof of (4.5).

Let us now bound summands. By (3.12),

$$
\begin{aligned}
\left|\int_{\mathfrak{e}_{i}} S^{c} \tilde{\boldsymbol{v}} \cdot \boldsymbol{t} d s\right| & =\left|\int_{D_{\boldsymbol{a}_{j}}^{h}} \int_{D_{\boldsymbol{a}_{k}}^{h}} \kappa_{j k} \int_{0}^{1} \tilde{\boldsymbol{v}}\left((1-s) \boldsymbol{y}_{j}+s \boldsymbol{y}_{k}\right) \cdot\left(\boldsymbol{y}_{k}-\boldsymbol{y}_{j}\right) d s d \boldsymbol{y}_{k} d \boldsymbol{y}_{j}\right| \\
& \leqslant C h \int_{D_{\boldsymbol{a}_{j}}^{h}} \int_{D_{\boldsymbol{a}_{k}}^{h}}\left|\kappa_{j k}\right| \int_{0}^{1}\left|\tilde{\boldsymbol{v}}\left((1-s) \boldsymbol{y}_{j}+s \boldsymbol{y}_{k}\right)\right| d s d \boldsymbol{y}_{k} d \boldsymbol{y}_{j} \\
& \leqslant C h \int_{D_{\boldsymbol{a}_{j}}^{h}} \int_{D_{\boldsymbol{a}_{k}}^{h}}\left|\kappa_{j k}\right|\left(\int_{0}^{1 / 2}+\int_{1 / 2}^{1}\right)\left|\tilde{\boldsymbol{v}}\left((1-s) \boldsymbol{y}_{j}+s \boldsymbol{y}_{k}\right)\right| d s d \boldsymbol{y}_{k} d \boldsymbol{y}_{j}=A+B
\end{aligned}
$$

Here we have broken the integral with respect to $s$ into two integrals, one over $s \in[0,1 / 2]$ (named $A$ ) and the other over $s \in[1 / 2,1]$ (named $B$ ).

The first can be bounded as follows:

$$
\begin{aligned}
& A:=\int_{D_{\boldsymbol{a}_{k}}^{h}} h\left|\kappa_{k}\right| \int_{0}^{1 / 2} \int_{D_{\boldsymbol{a}_{j}}^{h}}\left|\kappa_{j}\right|\left|\tilde{\boldsymbol{v}}\left((1-s) \boldsymbol{y}_{j}+s \boldsymbol{y}_{k}\right)\right| d \boldsymbol{y}_{j} d s d \boldsymbol{y}_{k} \\
& \left.\quad \leqslant \int_{D_{\boldsymbol{a}_{k}}^{h}} h\left|\kappa_{k}\right| \int_{0}^{1 / 2}\left\|\eta_{\boldsymbol{a}_{j}}\right\|_{L_{r}^{2}\left(D_{\boldsymbol{a}_{j}}^{h}\right)} \| \tilde{\boldsymbol{v}}\left((1-s) \boldsymbol{y}_{j}+s \boldsymbol{y}_{k}\right)\right) \|_{L_{r}^{2}\left(D_{\boldsymbol{a}_{j}}^{h}\right)} d s d \boldsymbol{y}_{k} .
\end{aligned}
$$

Now, consider the change of variable $\boldsymbol{z}=(1-s) \boldsymbol{y}_{j}+s \boldsymbol{y}_{k}$. Whenever $0 \leqslant s \leqslant \frac{1}{2}$, we have

$$
r\left(\boldsymbol{y}_{j}\right) \leqslant 2(1-s) r\left(\boldsymbol{y}_{j}\right) \leqslant 2\left((1-s) r\left(\boldsymbol{y}_{j}\right)+s r\left(\boldsymbol{y}_{k}\right)\right)=2 r(\boldsymbol{z}) .
$$

Hence, by Cauchy-Schwarz inequality,

$$
\begin{aligned}
\left.\int_{0}^{1 / 2} \| \tilde{\boldsymbol{v}}\left((1-s) \boldsymbol{y}_{j}+s \boldsymbol{y}_{k}\right)\right) \|_{L_{r}^{2}\left(D_{\boldsymbol{a}_{j}}^{h}\right)} d s & \left.=\left.\int_{0}^{1 / 2}\left(\int_{D_{\boldsymbol{a}_{j}}^{h}} r\left(\boldsymbol{y}_{j}\right) \mid \tilde{\boldsymbol{v}}\left((1-s) \boldsymbol{y}_{j}+s \boldsymbol{y}_{k}\right)\right)\right|^{2} d \boldsymbol{y}_{j}\right)^{1 / 2} d s \\
& \leqslant C\left(\int_{0}^{1 / 2} \int_{Z_{k j}} r(\boldsymbol{z})|\tilde{\boldsymbol{v}}(\boldsymbol{z})|^{2} \frac{d \boldsymbol{z}}{(1-s)^{2}} d s\right)^{1 / 2}
\end{aligned}
$$

where $Z_{k j}$ is the transformed domain under the change of variable - see Figure 3. Clearly, $(1-s)^{-2} \leqslant 4$ and $Z_{k j} \subset C_{K}$. Therefore, continuing from (4.13) and using (3.2),

$$
A \leqslant \frac{C h}{\sqrt{(h \delta)^{2} r_{\boldsymbol{a}_{j}}}}\|\tilde{\boldsymbol{v}}\|_{L_{r}^{2}\left(C_{K}\right)}\left\|\eta_{\boldsymbol{a}_{k}}\right\|_{L_{r}^{1}\left(D_{\boldsymbol{a}_{k}}^{h}\right)} .
$$




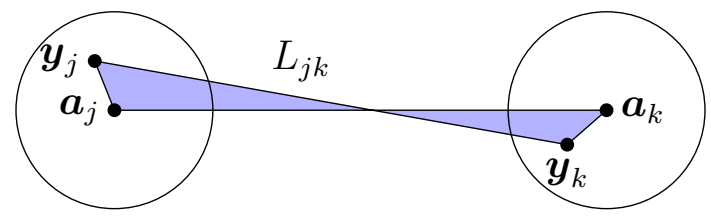

Figure 4. A domain of integration by parts in the proof of (4.6)

By (3.3),

$$
\left\|\eta_{\boldsymbol{a}_{k}}\right\|_{L_{r}^{1}\left(D_{\boldsymbol{a}_{k}}^{h}\right)} \leqslant C\left(1+\frac{h \delta}{r_{\boldsymbol{a}_{k}}}\right) \leqslant C,
$$

where we have also used (3.4). Hence

$$
A \leqslant \frac{C}{\delta \sqrt{r_{\boldsymbol{a}_{j}}}}\|\tilde{\boldsymbol{v}}\|_{L_{r}^{2}\left(C_{K}\right)} .
$$

By a similar argument, we can bound the other integral $B$ as well. Thus, returning to $(4.12)$, we have

$$
\left\|R_{h}^{c} \boldsymbol{v}\right\|_{L_{r}^{2}(K)}^{2} \leqslant \frac{C}{\delta^{2}}\left(\frac{r_{K}}{r_{a_{j}}}+\frac{r_{K}}{r_{a_{k}}}\right)\|\tilde{\boldsymbol{v}}\|_{L_{r}^{2}\left(C_{K}\right)}^{2} .
$$

Estimating the ratios as before - see (4.9) - we prove (4.11).

Proof of (4.6). To perform a similar argument leading to (4.6), we now need to bound $\left|\int_{\mathfrak{e}_{1}}\left(S^{c} \tilde{\boldsymbol{v}}_{h}-\boldsymbol{v}_{h}\right) \cdot \boldsymbol{t} d s\right|$. To this end, we will use an integration by parts over the area $L_{j k}$ enclosed by the line segments $\left[\boldsymbol{a}_{j}, \boldsymbol{a}_{k}\right],\left[\boldsymbol{a}_{k}, \boldsymbol{y}_{k}\right],\left[\boldsymbol{y}_{k}, \boldsymbol{y}_{j}\right]$ and $\left[\boldsymbol{y}_{j}, \boldsymbol{a}_{j}\right]$ (see Figure 4 ), namely

$$
\left|\int_{L} \operatorname{curl}_{r z} \tilde{\boldsymbol{v}}_{h} d \boldsymbol{x}\right|=\left|\int_{\left[\boldsymbol{a}_{j}, \boldsymbol{a}_{k}\right]+\left[\boldsymbol{a}_{k}, \boldsymbol{y}_{k}\right]+\left[\boldsymbol{y}_{k}, \boldsymbol{y}_{j}\right]+\left[\boldsymbol{y}_{j}, \boldsymbol{a}_{j}\right]} \tilde{\boldsymbol{v}}_{h} \cdot \boldsymbol{t} d s\right| .
$$

Beginning with (3.12) and using the above,

$$
\begin{aligned}
\left|\int_{\mathfrak{e}_{i}}\left(S^{c} \tilde{\boldsymbol{v}}_{h}-\boldsymbol{v}_{h}\right) \cdot \boldsymbol{t} d s\right| & =\left|\int_{D_{\boldsymbol{a}_{j}}^{h}} \int_{D_{\boldsymbol{a}_{k}}^{h}} \kappa_{j k}\left(\int_{\left[\boldsymbol{y}_{j}, \boldsymbol{y}_{k}\right]} \tilde{\boldsymbol{v}}_{h} \cdot \boldsymbol{t} d s-\int_{\left[\boldsymbol{a}_{j}, \boldsymbol{a}_{k}\right]} \boldsymbol{v}_{h} \cdot \boldsymbol{t} d s\right) d \boldsymbol{y}_{k} d \boldsymbol{y}_{j}\right| \\
& \leqslant \int_{D_{\boldsymbol{a}_{j}}^{h}} \int_{D_{\boldsymbol{a}_{k}}^{h}}\left|\kappa_{j k}\right|\left(\left|\int_{L_{j k}} \operatorname{curl}_{r z} \tilde{\boldsymbol{v}}_{h} d \boldsymbol{x}\right|+\left|\int_{\left[\boldsymbol{a}_{j}, \boldsymbol{y}_{j}\right]} \tilde{\boldsymbol{v}}_{h} \cdot \boldsymbol{t} d s\right|\right. \\
& \left.+\left|\int_{\left[\boldsymbol{a}_{k}, \boldsymbol{y}_{k}\right]} \tilde{\boldsymbol{v}}_{h} \cdot \boldsymbol{t} d s\right|\right) d \boldsymbol{y}_{k} d \boldsymbol{y}_{2} \\
& \leqslant \int_{D_{\boldsymbol{a}_{j}}^{h}} \int_{D_{\boldsymbol{a}_{k}}^{h}}\left|\kappa_{j k}\right| C h \delta\left(h\left\|\operatorname{curl}_{r z} \tilde{\boldsymbol{v}}_{h}\right\|_{L^{\infty}\left(L_{j k}\right)}+\left\|\tilde{\boldsymbol{v}}_{h}\right\|_{L^{\infty}\left(C_{K}\right)}\right) d \boldsymbol{y}_{k} d \boldsymbol{y}_{j},
\end{aligned}
$$

where we have used that $\left|L_{j k}\right| \leqslant C h(h \delta),\left|\left[\boldsymbol{a}_{j}, \boldsymbol{y}_{j}\right]\right| \leqslant C h \delta$, and $\left|\left[\boldsymbol{a}_{k}, \boldsymbol{y}_{k}\right]\right| \leqslant C h \delta$. Thus,

$$
\left|\int_{\mathfrak{e}_{i}}\left(S^{c} \tilde{\boldsymbol{v}}_{h}-\boldsymbol{v}_{h}\right) \cdot \boldsymbol{t} d s\right| \leqslant C h \delta\left(h\left\|\operatorname{curl}_{r z} \tilde{\boldsymbol{v}}_{h}\right\|_{L^{\infty}\left(C_{K}\right)}+\left\|\tilde{\boldsymbol{v}}_{h}\right\|_{L^{\infty}\left(C_{K}\right)}\right)\left\|\eta_{\boldsymbol{a}_{j}}\right\|_{L_{r}^{1}\left(D_{\boldsymbol{a}_{j}}^{h}\right)}\left\|\eta_{\boldsymbol{a}_{k}}\right\|_{L_{r}^{1}\left(D_{\boldsymbol{a}_{k}}^{h}\right)} .
$$




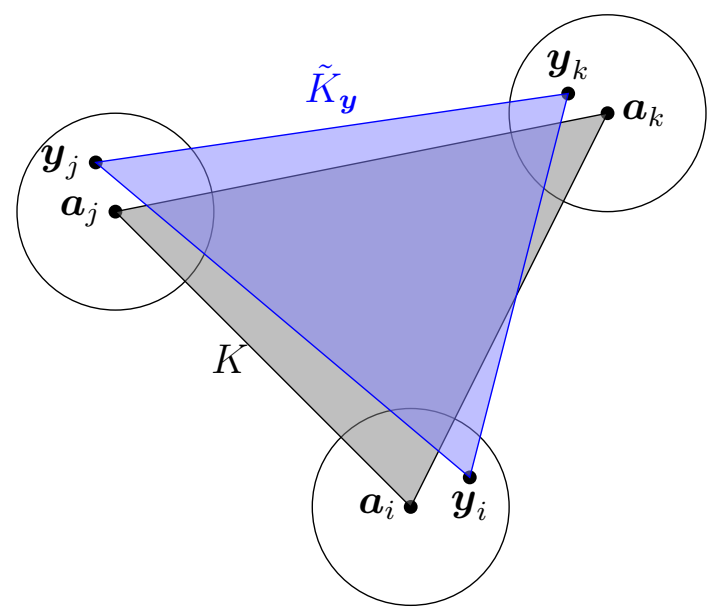

FiguRE $5 . K$ is mapped to $\tilde{K}_{\boldsymbol{y}}$ under the map $\boldsymbol{x} \mapsto \tilde{\boldsymbol{x}}_{\boldsymbol{y}}$ for each choice of $\left\{\boldsymbol{y}_{\ell}\right\}$.

The $L_{r}^{1}$-norms above are uniformly bounded - see (4.14). Hence, using Proposition 2.1 and proceeding as in (4.12), we reach

$$
\left\|R_{h}^{c} \boldsymbol{v}_{h}-\boldsymbol{v}_{h}\right\|_{L_{r}^{2}(K)}^{2} \leqslant C r_{K} h^{2} \delta^{2}\left\|\tilde{\boldsymbol{v}}_{h}\right\|_{L^{\infty}\left(C_{K}\right)}^{2} \leqslant C \delta^{2}\left\|\tilde{\boldsymbol{v}}_{h}\right\|_{L_{r}^{2}\left(C_{K}\right)}^{2},
$$

thus completing the proof of (4.6).

Proof of (4.7). Let

$$
J=\operatorname{det}\left(\frac{\boldsymbol{d} \tilde{\boldsymbol{x}}_{\boldsymbol{y}}}{\boldsymbol{d} \boldsymbol{x}}\right)=\left(\sum_{m=1}^{3}\left(\partial_{r} \lambda_{m}\right) \boldsymbol{y}_{m}\right) \times\left(\sum_{n=1}^{3}\left(\partial_{z} \lambda_{n}\right) \boldsymbol{y}_{n}\right)
$$

be the Jacobian appearing in the definition of $S^{o}$ - see (3.8). Let $\tilde{K}_{\boldsymbol{y}}$ denote the image of $K$ under the map $\boldsymbol{x} \mapsto \boldsymbol{x}_{\boldsymbol{y}}$ (see Figure 5). Obviously,

$$
|J|=\frac{\left|\tilde{K}_{y}\right|}{|K|} \leqslant C
$$

Then, by (2.6),

$$
\begin{aligned}
\left\|R_{h}^{o} w\right\|_{L_{r}^{2}(K)}^{2} & =\left\|I_{h}^{o} S^{o} \tilde{w}\right\|_{L_{r}^{2}(K)}^{2}=\int_{K} r(\boldsymbol{y})\left|\phi_{K} \frac{1}{|K|} \int_{K} S^{o} \tilde{w} d \boldsymbol{x}\right|^{2} d \boldsymbol{y} \\
& \leqslant C \frac{r_{K}}{h_{K}^{2}}\left|\int_{K} S^{o} \tilde{w} d \boldsymbol{x}\right|^{2},
\end{aligned}
$$

so now we proceed to estimate the last term. Let $T_{\ell}=\left\{\boldsymbol{x} \in K: \lambda_{\ell}(\boldsymbol{x})>\frac{1}{3}\right\}$, for $1 \leqslant \ell \leqslant 3$. Then we may overestimate the integral by

$$
\int_{K} S^{o} \tilde{w} d \boldsymbol{x} \leqslant \sum_{\ell=1}^{3}\left|\int_{T_{\ell}} S^{o} \tilde{w} d \boldsymbol{x}\right|
$$


We will now bound the first summand (and the others will be similarly bounded). By Fubini's theorem and Cauchy-Schwarz inequality,

$$
\begin{aligned}
\left|\int_{T_{1}} S^{o} \tilde{w} d \boldsymbol{x}\right| & \leqslant \int_{T_{1}} \int_{D_{a_{1}}^{h}} \int_{D_{\boldsymbol{a}_{2}}^{h}} \int_{D_{\boldsymbol{a}_{3}}^{h}}\left|\kappa_{123} J\right|\left|w\left(\tilde{\boldsymbol{x}}_{\boldsymbol{y}}\right)\right| d \boldsymbol{y}_{3} d \boldsymbol{y}_{2} d \boldsymbol{y}_{1} d \boldsymbol{x} \\
& =\int_{D_{\boldsymbol{a}_{2}}^{h}} \int_{D_{\boldsymbol{a}_{3}}^{h}}\left|\kappa_{23}\right|\left(\int_{T_{1}} \int_{D_{\boldsymbol{a}_{1}}^{h}} r\left(\boldsymbol{y}_{1}\right)\left|\eta_{\boldsymbol{a}_{1}}\left(\boldsymbol{y}_{1}\right) w\left(\tilde{\boldsymbol{x}}_{\boldsymbol{y}}\right) J\right| d \boldsymbol{y}_{1} d \boldsymbol{x}\right) d \boldsymbol{y}_{3} d \boldsymbol{y}_{2} \\
& \leqslant\left\|\eta_{\boldsymbol{a}_{1}}\right\|_{L_{r}^{2}\left(D_{\boldsymbol{a}_{1}}^{h}\right)} \sqrt{\left|T_{1}\right||J|} \int_{D_{\boldsymbol{a}_{2}}^{h}} \int_{D_{\boldsymbol{a}_{3}}^{h}}\left|\kappa_{23}\right|\left(\int_{T_{1}} \int_{D_{\boldsymbol{a}_{1}}^{h}} r\left(\boldsymbol{y}_{1}\right)\left|w\left(\tilde{\boldsymbol{x}}_{\boldsymbol{y}}\right)\right|^{2}|J| d \boldsymbol{y}_{1} d \boldsymbol{x}\right)^{1 / 2} d \boldsymbol{y}_{3} d \boldsymbol{y}_{2}
\end{aligned}
$$

To estimate the integral in parenthesis, we first observe that since $1 / \lambda_{1}(\boldsymbol{x})<3$ for all $\boldsymbol{x} \in T_{1}$, the inequality

$$
\begin{aligned}
r\left(\boldsymbol{y}_{1}\right)=\frac{1}{\lambda_{1}(\boldsymbol{x})} \lambda_{1}(\boldsymbol{x}) r\left(\boldsymbol{y}_{1}\right) & <3 r\left(\lambda_{1}(\boldsymbol{x}) \boldsymbol{y}_{1}\right) \\
& \leqslant 3\left(r\left(\lambda_{1}(\boldsymbol{x}) \boldsymbol{y}_{1}\right)+r\left(\lambda_{2}(\boldsymbol{x}) \boldsymbol{y}_{2}\right)+r\left(\lambda_{3}(\boldsymbol{x}) \boldsymbol{y}_{3}\right)\right)=3 r\left(\tilde{\boldsymbol{x}}_{\boldsymbol{y}}\right)
\end{aligned}
$$

holds, so

$$
\begin{aligned}
\int_{T_{1}} \int_{D_{a_{1}}^{h}} r\left(\boldsymbol{y}_{1}\right)\left|w\left(\tilde{\boldsymbol{x}}_{\boldsymbol{y}}\right)\right|^{2}|J| d \boldsymbol{y}_{1} d \boldsymbol{x} & \leqslant C \int_{T_{1}} \int_{D_{\boldsymbol{a}_{1}}^{h}} r\left(\tilde{\boldsymbol{x}}_{\boldsymbol{y}}\right)\left|w\left(\tilde{\boldsymbol{x}}_{\boldsymbol{y}}\right)\right|^{2}|J| d \boldsymbol{y}_{1} d \boldsymbol{x} \\
& =C \int_{D_{\boldsymbol{a}_{1}}^{h}} \int_{\tilde{T}_{1}} r(\boldsymbol{z})|w(\boldsymbol{z})|^{2} d \boldsymbol{z} d \boldsymbol{y}_{1} \\
& \leqslant C(h \delta)^{2}\|w\|_{L_{r}^{2}\left(C_{K}\right)}^{2},
\end{aligned}
$$

where $\tilde{T}_{1}$ is the image of $T_{1}$ under the map $\boldsymbol{x} \mapsto \tilde{\boldsymbol{x}}_{\boldsymbol{y}}$ and we have used the bound for its Jacobian in (4.16). Thus, returning to (4.19), using the above estimate together with Proposition 3.1,

$$
\left|\int_{T_{1}} S^{o} \tilde{w} d \boldsymbol{x}\right| \leqslant \frac{C}{\sqrt{(h \delta)^{2} r_{\boldsymbol{a}_{1}}}} h(h \delta)\|w\|_{L_{r}^{2}\left(C_{K}\right)} \int_{D_{\boldsymbol{a}_{2}}^{h}} \int_{D_{\boldsymbol{a}_{3}}^{h}}\left|\kappa_{23}\right| d \boldsymbol{y}_{3} d \boldsymbol{y}_{2} .
$$

Due to (4.14), this estimate simplifies to

$$
\left|\int_{T_{1}} S^{o} \tilde{w} d \boldsymbol{x}\right| \leqslant \frac{C h}{\sqrt{r_{a_{1}}}}\|w\|_{L_{r}^{2}\left(C_{K}\right)}
$$

A similar estimate holds for all the three integrals in (4.18).

Therefore, returning to (4.17) we find that

$$
\left\|R_{h}^{o} w\right\|_{L_{r}^{2}(K)}^{2} \leqslant C\left(\frac{r_{K}}{r_{\boldsymbol{a}_{1}}}+\frac{r_{K}}{r_{\boldsymbol{a}_{2}}}+\frac{r_{K}}{r_{\boldsymbol{a}_{3}}}\right)\|\boldsymbol{w}\|_{L_{r}^{2}\left(C_{K}\right)}^{2}
$$

and the proof is finished by the estimate (4.9). 
Proof of (4.8). On the element $K$, by virtue of (3.1), we can write

$$
\begin{aligned}
\left.\left(R_{h}^{o} w_{h}-w_{h}\right)\right|_{K} & =\frac{1}{|K|} \int_{K} \int_{D_{\boldsymbol{a}_{1}}^{h}} \int_{D_{\boldsymbol{a}_{2}}^{h}} \int_{D_{\boldsymbol{a}_{3}}^{h}} \kappa_{123}\left(J w_{h}\left(\tilde{\boldsymbol{x}}_{\boldsymbol{y}}\right)-w_{h}(\boldsymbol{x})\right) d \boldsymbol{y}_{3} d \boldsymbol{y}_{2} d \boldsymbol{y}_{1} d \boldsymbol{x} \\
& =\frac{1}{|K|} \int_{D_{\boldsymbol{a}_{1}}^{h}} \int_{D_{\boldsymbol{a}_{2}}^{h}} \int_{D_{\boldsymbol{a}_{3}}^{h}} \kappa_{123}\left(\int_{\tilde{K}_{\boldsymbol{y}}} w_{h}(\boldsymbol{z}) d \boldsymbol{z}-\int_{K} w_{h}(\boldsymbol{x}) d \boldsymbol{x}\right) d \boldsymbol{y}_{3} d \boldsymbol{y}_{2} d \boldsymbol{y}_{1}
\end{aligned}
$$

by a change of variables. The difference of the integrals within the parenthesis can be written as integrals over small domains. Indeed,

$$
\begin{aligned}
\int_{\tilde{K}_{\boldsymbol{y}}} w_{h}(\boldsymbol{z}) d \boldsymbol{z}-\int_{K} w_{h}(\boldsymbol{x}) d \boldsymbol{x} & =\int_{\tilde{K}_{\boldsymbol{y}} \cap K}\left(w_{h}(\boldsymbol{z})-w_{h}(\boldsymbol{z})\right) d \boldsymbol{z} \\
& +\int_{\tilde{K}_{\boldsymbol{y}} \backslash K} w_{h}(\boldsymbol{z}) d \boldsymbol{z}-\int_{K \backslash \tilde{K}_{\boldsymbol{y}}} w_{h}(\boldsymbol{x}) d \boldsymbol{x},
\end{aligned}
$$

where the first integral on the right hand side vanishes, and the remaining can be bounded using $\left|\left(\tilde{K}_{\boldsymbol{y}} \backslash K\right) \cup\left(K \backslash \tilde{K}_{\boldsymbol{y}}\right)\right| \leqslant C h(h \delta)$ - see Figure 5. Thus,

$$
\begin{aligned}
\left\|R_{h}^{o} w_{h}-w_{h}\right\|_{L_{r}^{2}(K)}^{2} & \leqslant \frac{C r_{K}}{|K|^{2}} h^{2}(h \delta)^{2}\left\|\tilde{w}_{h}\right\|_{L^{\infty}\left(C_{K}\right)}^{2} \\
& \leqslant C \delta^{2}\left\|\tilde{w}_{h}\right\|_{L_{r}^{2}(K)}^{2}
\end{aligned}
$$

by Proposition 2.1. This proves the last estimate (4.8).

\section{The MAin RESUlt}

In this section we state and prove our main theorem on the existence of the commuting projectors bounded in the weighted $L_{r}^{2}$-norms. As already mentioned, the quasiinterpolation operators obtained in the previous section are not projectors. So in this section, we modify these operators to obtain projections. The definitions of the final projections appear below in Definition 5.1 and the main result is Theorem 5.1.

The basic idea, again due to [26], stems from the observation that each of the operators $R_{h}^{g}, R_{h}^{c}$ and $R_{h}^{o}$, when restricted to their respective range finite element spaces $\left(V_{h, \triangleright}, \boldsymbol{W}_{h, \triangleright}, S_{h}\right.$, resp.) are invertible for small $\delta$.

Lemma 5.1. There are operators

$$
J_{h}^{g}: V_{h, \triangleright} \rightarrow V_{h, \triangleright}, \quad J_{h}^{c}: \boldsymbol{W}_{h, \triangleright} \rightarrow \boldsymbol{W}_{h, \triangleright}, \quad J_{h}^{o}: S_{h} \rightarrow S_{h},
$$

and $a \delta_{1}>0$ such that for all $0<\delta<\delta_{1}$, the operators $\left.R_{h}^{g}\right|_{V_{h, \triangleright}},\left.R_{h}^{c}\right|_{W_{h, \triangleright}}$, and $\left.R_{h}^{o}\right|_{S_{h}}$ are invertible, their inverses are $J_{h}^{g}, J_{h}^{c}$, and $J_{h}^{o}$, resp., and their operator norms satisfy

$$
\left\|J_{h}^{g}\right\|_{L_{r}^{2}(D)} \leqslant 2, \quad\left\|J_{h}^{c}\right\|_{L_{r}^{2}(D)} \leqslant 2, \quad\left\|J_{h}^{o}\right\|_{L_{r}^{2}(D)} \leqslant 2 .
$$

Proof. Considering any one of the three operators, say $\left.R_{h}^{c}\right|_{\boldsymbol{W}_{h, \triangleright}}$, we note that by (4.6), there exists a $\delta_{1}$ such that the $L_{r}^{2}$-operator norm of $\left.\left(I-R_{h}^{c}\right)\right|_{\boldsymbol{W}_{h, \triangleright}}$ is less than $1 / 2$ for all $\delta<\delta_{1}$. Consequently, by a standard Neumann series argument, the series

$$
J_{h}^{c}:=\left(I-\left.\left(I-R_{h}^{c}\right)\right|_{\boldsymbol{W}_{h, \triangleright}}\right)^{-1}=\sum_{m=0}^{\infty}\left(I-\left.R_{h}^{c}\right|_{\boldsymbol{W}_{h, \triangleright}}\right)^{m},
$$


converges in $L_{r}^{2}(D)$-norm, the inverse $\left(\left.R_{h}^{c}\right|_{\boldsymbol{W}_{h, \triangleright}}\right)^{-1}=\left(I-\left.\left(I-R_{h}^{c}\right)\right|_{\boldsymbol{W}_{h, \triangleright}}\right)^{-1}$ exists (which we denote by $J_{h}^{c}$ ), and moreover the norm bound

$$
\left\|J_{h}^{c}\right\|_{L_{r}^{2}(D)} \leqslant \frac{1}{1-\left\|\left.\left(I-R_{h}^{c}\right)\right|_{\boldsymbol{W}_{h, \triangleright}}\right\|_{L_{r}^{2}(D)}} \leqslant \frac{1}{1-(1 / 2)}=2,
$$

holds. Similarly the other two inverses exist and the same bound holds.

For the rest of the paper, we fix a $\delta \in\left(0, \delta_{1}\right]$, where $\delta_{1}$ is as given by Lemma 5.1. From now on, we will let our generic constant $C$ depend on (this fixed) $\delta$. It will continue to remain independent of the mesh size $h$ and the functions being estimated. We can now give the final definition of the smoothed projectors.

Definition 5.1. Define $\Pi_{h}^{g}: L_{r}^{2}(D) \rightarrow V_{h, \triangleright}, \Pi_{h}^{c}: L_{r}^{2}(D)^{2} \rightarrow \boldsymbol{W}_{h, \triangleright}$, and $\Pi_{h}^{o}: L_{r}^{2}(D) \rightarrow S_{h}$ by

$$
\Pi_{h}^{g}=J_{h}^{g} R_{h}^{g}, \quad \Pi_{h}^{c}=J_{h}^{c} R_{h}^{c}, \quad \Pi_{h}^{o}=J_{h}^{o} R_{h}^{o} .
$$

Theorem 5.1. The above operators are projectors and have the following properties:

(1) Continuity. There exists a $C>0$ such that

$$
\begin{array}{lll}
\left\|\Pi_{h}^{g} u\right\|_{L_{r}^{2}(D)} \leqslant C\|u\|_{L_{r}^{2}(D)}, & & \forall u \in L_{r}^{2}(D), \\
\left\|\Pi_{h}^{c} \boldsymbol{v}\right\|_{L_{r}^{2}(D)} \leqslant C\|\boldsymbol{v}\|_{L_{r}^{2}(D)}, & & \forall \boldsymbol{v} \in L_{r}^{2}(D)^{2}, \\
\left\|\Pi_{h}^{o} w\right\|_{L_{r}^{2}(D)} \leqslant C\|w\|_{L_{r}^{2}(D)}, & & \forall w \in L_{r}^{2}(D) .
\end{array}
$$

(2) Commutativity. The operators satisfy the following commuting diagram:

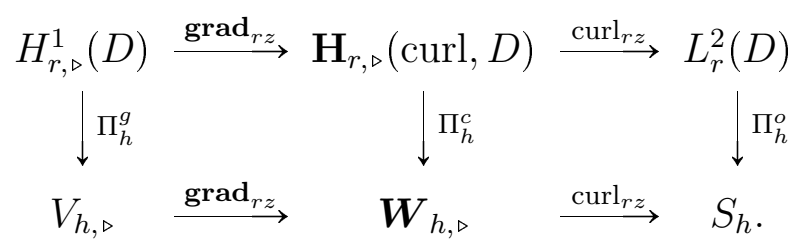

(3) Approximation. For all $0 \leqslant s \leqslant 1$,

(a) $\left\|u-\Pi_{h}^{g} u\right\|_{r} \leqslant C h^{s}\|u\|_{H_{r}^{s}(D)}$ for all $u \in H_{r}^{s}(D)$.

(b) $\left\|\boldsymbol{v}-\Pi_{h}^{c} \boldsymbol{v}\right\|_{r} \leqslant C h^{s}\|\boldsymbol{v}\|_{H_{r}^{s}(D)}$ for all $\boldsymbol{v} \in H_{r}^{s}(D)^{2}$.

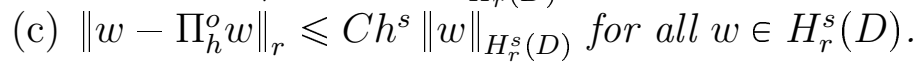

Proof. First, to verify that the operators are projectors, we observe that by Lemma 5.1,

$$
\left(\Pi_{h}^{g}\right)^{2} u=J_{h}^{g} R_{h}^{g} J_{h}^{g} R_{h}^{g} u=J_{h}^{g}\left(\left.R_{h}^{g}\right|_{V_{h, \triangleright}} J_{h}^{g}\right) R_{h}^{g} u=J_{h}^{g} R_{h}^{g} u=\Pi_{h}^{g} u,
$$

so $\Pi_{h}^{g}$ is a projector. Similarly, the other two operators are also projectors.

The continuity estimates follow from the estimates of Lemma 4.2 and Lemma 5.1. For instance, $\left\|\Pi_{h}^{c}\right\|_{L_{r}^{2}(D)} \leqslant\left\|J_{h}^{c}\right\|_{L_{r}^{2}(D)}\left\|R_{h}^{c}\right\|_{L_{r}^{2}(D)} \leqslant 2 C \delta^{-3 / 2}$.

The commutativity identities follow from the commutativity properties of the $R_{h^{-}}$ operators stated in Lemma 4.1 and those of the $J_{h}$-operators. The latter follows from the former. For example, let $J_{h}^{c} \boldsymbol{v}_{h}=\boldsymbol{w}_{h}$ for $\boldsymbol{v}_{h}, \boldsymbol{w}_{h} \in \boldsymbol{W}_{h, \triangleright}$. Then, $R_{h}^{c} \boldsymbol{w}_{h}=\boldsymbol{v}_{h}$ and $\operatorname{curl}_{r z} \boldsymbol{v}_{h}=\operatorname{curl}_{r z}\left(R_{h}^{c} \boldsymbol{w}_{h}\right)=R_{h}^{o} \operatorname{curl}_{r z} \boldsymbol{w}_{h}$. Thus,

$$
J_{h}^{o}\left(\operatorname{curl}_{r z} \boldsymbol{v}_{h}\right)=J_{h}^{o}\left(R_{h}^{o} \operatorname{curl}_{r z} \boldsymbol{w}_{h}\right)=\operatorname{curl}_{r z} \boldsymbol{w}_{h}=\operatorname{curl}_{r z}\left(J_{h}^{c} \boldsymbol{v}_{h}\right) \text {, }
$$


for all $\boldsymbol{v}_{h} \in \boldsymbol{W}_{h, \triangleright}$. Hence,

$$
\begin{aligned}
\operatorname{curl}_{r z}\left(\Pi_{h}^{c} \boldsymbol{v}\right) & =\operatorname{curl}_{r z}\left(J_{h}^{c} R_{h}^{c} \boldsymbol{v}\right)=J_{h}^{o} \operatorname{curl}_{r z}\left(R_{h}^{c} \boldsymbol{v}_{h}\right) & & \text { by }(5.3) \\
& =J_{h}^{o} R_{h}^{o} \operatorname{curl}_{r z} \boldsymbol{v}_{h} & & \text { by Lemma 4.1. } \\
& =\Pi_{h}^{o} \operatorname{curl}_{r z} \boldsymbol{v}_{h} . & &
\end{aligned}
$$

This proves the commutativity property in the right end of the diagram (5.2). The remaining identities are proved similarly.

To prove the approximation estimates, consider a general $\boldsymbol{v}_{h} \in \boldsymbol{W}_{h, \triangleright}$. Since $\Pi_{h}^{c}$ is a projection $\Pi_{h}^{c} \boldsymbol{v}_{h}=\boldsymbol{v}_{h}$. Hence,

$$
\begin{aligned}
\left\|\boldsymbol{v}-\Pi_{h}^{c} \boldsymbol{v}\right\|_{L_{r}^{2}(D)} & =\left\|\left(\boldsymbol{v}-\boldsymbol{v}_{h}\right)-\Pi_{h}^{c}\left(\boldsymbol{v}-\boldsymbol{v}_{h}\right)\right\|_{L_{r}^{2}(D)} \\
& \leqslant(1+C)\left\|\boldsymbol{v}-\boldsymbol{v}_{h}\right\|_{L_{r}^{2}(D)}
\end{aligned}
$$

for any $\boldsymbol{v}_{h} \in \boldsymbol{W}_{h, \triangleright}$. Hence taking the infimum over $\boldsymbol{v}_{h}$,

$$
\left\|\boldsymbol{v}-\Pi_{h}^{c} \boldsymbol{v}\right\|_{L_{r}^{2}(D)} \leqslant C \inf _{\boldsymbol{v}_{h} \in \boldsymbol{W}_{h, \triangleright}}\left\|\boldsymbol{v}-\boldsymbol{v}_{h}\right\|_{L_{r}^{2}(D)} .
$$

Similar inequalities hold for the other two projectors as well.

It remains to bound the best approximation error using approximants with known convergence rates in the weighted norms. In the case of $S_{h}$, this is standard (see e.g., [4, Lemma 5]). In the case of $\boldsymbol{W}_{h, \triangleright}$ and $V_{h, \triangleright}$, this follows from [12]. Namely, the interpolants constructed in [12, Lemma 5.3] show that for all $\boldsymbol{v} \in H_{r}^{1}(D)^{2}$ and $u \in H_{r}^{1}(D)$, there are $\boldsymbol{v}_{h} \in \boldsymbol{W}_{h, \triangleright}$ and $u_{h} \in V_{h, \triangleright}$ such that

$$
\begin{aligned}
\left\|\boldsymbol{v}-\boldsymbol{v}_{h}\right\|_{L_{r}^{2}(D)} & \leqslant C h|\boldsymbol{v}|_{H_{r}^{1}(D)}, \\
\left\|u-u_{h}\right\|_{L_{r}^{2}(D)} & \leqslant C h|u|_{H_{r}^{1}(D)} .
\end{aligned}
$$

Thus the estimates of item (3) are proved for the case $s=1$. The same estimates in the case $s=0$ trivially follow item (1). For all intermediate values of $s$, the estimates follow by the standard theory of interpolation of operators [5].

Remark 5.1. We considered the weight function $r$ because of its many applications in axisymmetric problems. But the techniques are generalizable to handle other weight functions. The crucial estimates are those of Lemma 4.2. One would need to generalize them to the case of the particular weight function of interest.

Remark 5.2. The quasi-interpolants of the previous section themselves have approximation properties (even though they are not projectors). It may be possible to quantify these properties in the higher order case by varying $\ell$ in Proposition 3.1. But note that in our analysis we have only used the $\ell=0$ case of the proposition.

\section{Application to axisymmetric Maxwell equations}

In this section, we will use the commuting projectors of Section 5 to prove a convergence result for the edge finite element approximation of the so-called "meridian" subproblem of the axisymmetric Maxwell system. The three-dimensional (3D) time harmonic Maxwell equations decouples into two two-dimensional (2D) problems: one called the azimuthal problem and the other the meridian problem [3, 12]. The meridian problem is posed on 
the right half of the $r z$-plane (sometimes called the meridian half-plane). It finds the $r$ and $z$ components of the electric field, i.e., $\boldsymbol{E}_{r z}=E_{r} \boldsymbol{e}_{r}+E_{z} \boldsymbol{e}_{z}$. The components $E_{r}$ and $E_{z}$ are functions of $r$ and $z$ alone (as there is no $\theta$ dependence due to axial symmetry).

The meridian problem is to find $\boldsymbol{E}_{r z}$ satisfying

$$
\operatorname{curl}_{r z}\left(\frac{1}{\mu} \operatorname{curl}_{r z} \boldsymbol{E}_{r z}\right)-\kappa^{2} \epsilon \boldsymbol{E}_{r z}=\boldsymbol{F},
$$

where the scalar-valued $\operatorname{curl}_{r z}$ is as defined in (2.1), the vector-valued curl is defined by

$$
\operatorname{curl}_{r z} \psi=\left(-\partial_{z} \psi, r^{-1} \partial_{r}(r \psi)\right),
$$

the material coefficient $\mu$ represents magnetic permeability, $\epsilon$ is the dielectric constant, $\boldsymbol{F}$ represents given sources, all of which are axisymmetric, and $\kappa$ is the wavenumber. Recall that $D \subset \mathbb{R}_{+}^{2}$ denotes the restriction of the original axisymmetric $3 D$ domain, which we will call $\Omega \subset \mathbb{R}^{3}$, to the meridian half-plane, i.e., $\Omega$ is obtained by rotating $D$ about the $z$-axis. Recall that we have assumed that $D$ has Lipschitz boundary, $\Gamma_{1}$ is connected, and $D$ is simply connected. This implies that $\partial \Omega$ is Lipschitz. We are able to perform the analysis with this minimal regularity on $\partial \Omega$ due to the low-regularity projectors constructed earlier.

Perfect electric boundary conditions on $\partial \Omega$ translates to the boundary condition $\boldsymbol{E}_{r z} \cdot \boldsymbol{t}=$ 0 on $\partial D$, where $\boldsymbol{t}$ denotes the unit tangent vector. For error analysis, we consider a model problem for real-valued functions with this boundary condition, together with unit material properties. Its weak formulation is to find $\boldsymbol{u} \in \boldsymbol{H}_{r, \triangleright}(\operatorname{curl}, D)$ such that

$$
A(\boldsymbol{u}, \boldsymbol{v})=(\boldsymbol{F}, \boldsymbol{v})_{r}, \quad \forall \boldsymbol{v} \in \boldsymbol{H}_{r, \triangleright}(\operatorname{curl}, D),
$$

where $A: \boldsymbol{H}_{r, \triangleright}(\operatorname{curl}, D) \times \boldsymbol{H}_{r, \triangleright}(\operatorname{curl}, D) \mapsto \mathbb{R}$ is the bilinear form defined by

$$
A(\boldsymbol{u}, \boldsymbol{v})=\left(\operatorname{curl}_{r z} \boldsymbol{u}, \operatorname{curl}_{r z} \boldsymbol{v}\right)_{r}-\kappa^{2}(\boldsymbol{u}, \boldsymbol{v})_{r} .
$$

There is a countable set of real values $\kappa$ for which (6.2) does not have a unique solution [20]. Throughout this paper, we will assume that $\kappa$ is not one of those Maxwell eigenvalues so that (6.2) is uniquely solvable.

The corresponding discrete problem is to find $\boldsymbol{u}_{h} \in \boldsymbol{W}_{h, \triangleright}$ such that

$$
A\left(\boldsymbol{u}_{h}, \boldsymbol{v}_{h}\right)=\left(\boldsymbol{F}, \boldsymbol{v}_{h}\right)_{r},
$$

for all $\boldsymbol{v}_{h} \in \boldsymbol{W}_{h, \triangleright}$. For brevity, let us denote the $\boldsymbol{H}_{r}(\operatorname{curl}, D)$-inner product by

$$
(\boldsymbol{u}, \boldsymbol{v})_{\Lambda}=\left(\operatorname{curl}_{r z} \boldsymbol{u}, \operatorname{curl}_{r z} \boldsymbol{v}\right)_{r}+(\boldsymbol{u}, \boldsymbol{v})_{r},
$$

and the corresponding norm by $\|\boldsymbol{v}\|_{\Lambda}=(\boldsymbol{v}, \boldsymbol{v})_{\Lambda}^{1 / 2}$. The following error estimate is the main new result we will prove in this section. Note that an error estimate for the positive definite problem (obtained by replacing $A(\cdot, \cdot)$ by $(\cdot, \cdot)_{\Lambda}$ in $(6.3)$ ) follows from the analysis in [12, Theorem 6.1]. However, the analysis of our indefinite problem is more involved.

Theorem 6.1 (Quasioptimality). Suppose (6.2) has a unique solution $\boldsymbol{u} \in \boldsymbol{H}_{r, \triangleright}(\mathrm{curl}, D)$. Then, there are constants $h_{0}$ and $C$ such that, for all $0<h<h_{0}$, (6.3) also has a unique solution $\boldsymbol{u}_{h}$, and

$$
\left\|\boldsymbol{u}-\boldsymbol{u}_{h}\right\|_{\Lambda} \leqslant C \inf _{\boldsymbol{w}_{h} \in \boldsymbol{W}_{h, \triangleright}}\left\|\boldsymbol{u}-\boldsymbol{w}_{h}\right\|_{\Lambda} .
$$


The constants $h_{0}$ and $C$ depend on $\kappa$ but are independent of $\boldsymbol{u}, \boldsymbol{u}_{h}$, and $h$.

Similar results are well known for the standard (unweighted) Maxwell system. One of the first such results for the time-harmonic 3D system was proved by Monk in [19] using a variation of the Schatz [24] duality argument. These techniques were refined and used in $[14,16]$ for preconditioning purposes using the new tools introduced in [1]. These works in turn prompted the development of a cleaner error analysis for the 3D Maxwell equations [21]. All these developments are summarized in the book $[20, \S 7.2]$ which also details what is now considered the standard proof of quasioptimality of edge element approximations of the 3D Maxwell system. The technique we will employ to prove Theorem 6.1 follows along the lines of this standard proof. We make a few further simplifications, possible due to the availability of the Schöberl projectors (constructed in the previous section). However, we need a few new ingredients to handle the additional complications resulting from our degenerate weight function. We begin with a regularity result for the meridian problem (6.2).

Lemma 6.1. Suppose $\boldsymbol{F} \in L_{r}^{2}(D)^{2}$ satisfies

$$
\left(\boldsymbol{F}, \operatorname{grad}_{r z} \phi\right)_{r}=0 \quad \forall \phi \in H_{r, \triangleright}^{1}(D),
$$

and suppose $\boldsymbol{u}$ solves (6.2). Then

$$
\|\boldsymbol{u}\|_{H_{r}^{\frac{1}{2}}(D)}+\left\|\operatorname{curl}_{r z} \boldsymbol{u}\right\|_{H_{r}^{\frac{1}{2}(D)}} \leqslant C\|\boldsymbol{F}\|_{r} .
$$

Proof. We use the available 3D regularity results. For any Sobolev space $X(\Omega)$, we will use $X(\Omega)$ to indicate its subspace of axisymmetric (scalar or vector) functions. Given a $2 \mathrm{D}$ vector field $\boldsymbol{v}(r, z)=\left(v_{r}(r, z), v_{z}(r, z)\right)$ on $\mathrm{D}$, we define its revolution as a $3 \mathrm{D}$ vector field $\boldsymbol{v}^{\Omega}(\boldsymbol{x})$ on $\Omega$ by $\boldsymbol{v}^{\Omega}(r, \theta, z)=v_{r}(r, z) \boldsymbol{e}_{r}+0 \boldsymbol{e}_{\theta}+v_{z}(r, z) \boldsymbol{e}_{z}$.

By the isomorphisms of axisymmetry in [3] (such as between $\breve{L}^{2}(\Omega)$ and $L_{r}^{2}(D)$ ), we know that $\boldsymbol{u}^{\Omega} \in \breve{\boldsymbol{H}}_{0}(\operatorname{curl}, \Omega)$ and $\boldsymbol{F}^{\Omega} \in \breve{L}^{2}(\Omega)^{2}$. Moreover, it was shown in [12] that condition (6.4) implies that $\left(\boldsymbol{F}^{\Omega}, \operatorname{grad} \zeta\right)_{r}=0$ for all $\zeta \in H_{0}^{1}(\Omega)$. Therefore, by taking derivative in the sense of distributions, $\left(\operatorname{div} \boldsymbol{F}^{\Omega}, \zeta\right)=0$ for all $\zeta \in \mathcal{D}(\Omega)$, and so $\operatorname{div} \boldsymbol{F}^{\Omega}=0$ in $L^{2}(\Omega)$. Similarly a direct calculation from (6.2) shows that curl curl $\boldsymbol{u}^{\Omega}-\kappa^{2} \boldsymbol{u}^{\Omega}=\boldsymbol{F}^{\Omega}$. All together, we have that $\boldsymbol{u}^{\Omega} \in \breve{\boldsymbol{H}}_{0}(\operatorname{curl}, \Omega) \cap \breve{H}(\operatorname{div}, \Omega)$. Therefore, since $\partial \Omega$ is Lipschitz, applying the $3 \mathrm{D}$ result of $[13$, Theorem 2$]$, we obtain

$$
\left\|\boldsymbol{u}^{\Omega}\right\|_{H^{\frac{1}{2}(\Omega)}}+\left\|\operatorname{curl} \boldsymbol{u}^{\Omega}\right\|_{H^{\frac{1}{2}(\Omega)}} \leqslant\left\|\boldsymbol{F}^{\Omega}\right\|_{L^{2}(\Omega)} .
$$

Now, it is known that [3] the spaces $\breve{\boldsymbol{H}}^{\frac{1}{2}}(\Omega)$ and $H_{r}^{\frac{1}{2}}(D) \times H_{r}^{\frac{1}{2}}(D) \times H_{r}^{\frac{1}{2}}(D)$ are isomorphic. Since the $\theta$-component of $\operatorname{curl} \boldsymbol{u}^{\Omega}$ is $\operatorname{curl}_{r z} \boldsymbol{u}$, this implies the stated result.

The next lemma concerns a "solenoidal" projection operator $\boldsymbol{S}$. For any $\boldsymbol{w}_{h} \in \boldsymbol{W}_{h, \triangleright}$ define $\boldsymbol{S} \boldsymbol{w}_{h} \in \boldsymbol{H}_{r, \triangleright}(\operatorname{curl}, D)$, together with $P \boldsymbol{w}_{h} \in L_{r}^{2}(D)$, as the unique solution of the dual mixed variational equations

$$
\begin{array}{rlrl}
\left(\boldsymbol{S} \boldsymbol{w}_{h}, \boldsymbol{v}\right)_{r}-( & \left(P \boldsymbol{w}_{h}, \operatorname{curl}_{r z} \boldsymbol{v}\right)_{r}=0, & & \forall \boldsymbol{v} \text { in } \boldsymbol{H}_{r, \triangleright}(\operatorname{curl}, D), \\
\left(s, \operatorname{curl}_{r z} \boldsymbol{S} \boldsymbol{w}_{h}\right)_{r}=\left(s, \operatorname{curl}_{r z} \boldsymbol{w}_{h}\right)_{r}, & \forall s \text { in } L_{r}^{2}(D) .
\end{array}
$$

(Note that setting $\boldsymbol{v}$ to gradients, we can conclude that $\boldsymbol{S} \boldsymbol{w}_{h}$ is solenoidal.) 
Lemma 6.2. Let $\boldsymbol{w}_{h} \in \boldsymbol{W}_{h, \triangleright}$ satisfy $\left(\boldsymbol{w}_{h}, \operatorname{grad}_{r z} \phi_{h}\right)_{r}=0$ for all $\phi_{h} \in V_{h, \triangleright}$. Then

$$
\left\|\boldsymbol{S} \boldsymbol{w}_{h}-\boldsymbol{w}_{h}\right\|_{r} \leqslant C h^{\frac{1}{2}}\left\|\operatorname{curl}_{r z} \boldsymbol{w}_{h}\right\|_{r} .
$$

Proof. By the exactness of the sequence (2.2), we know that $\boldsymbol{w}_{h}=\operatorname{curl}_{r z}^{\prime} p_{h}$, for some $p_{h} \in S_{h}$, where $\operatorname{curl}_{r z}^{\prime}: S_{h} \rightarrow \boldsymbol{W}_{h, \triangleright}$ denotes the $L_{r}^{2}$-adjoint of $\operatorname{curl}_{r z}: \boldsymbol{W}_{h, \triangleright} \rightarrow S_{h}$, i.e.,

$$
\left(\boldsymbol{w}_{h}, \boldsymbol{v}_{h}\right)_{r}-\left(p_{h}, \operatorname{curl}_{r z} \boldsymbol{v}_{h}\right)_{r}=0, \quad \forall \boldsymbol{v}_{h} \in \boldsymbol{W}_{h, \triangleright} .
$$

Subtracting this from (6.5), we obtain

$$
\left(\boldsymbol{S} \boldsymbol{w}_{h}-\boldsymbol{w}_{h}, \boldsymbol{v}_{h}\right)_{r}-\left(P \boldsymbol{w}_{h}-p_{h}, \operatorname{curl}_{r z} \boldsymbol{v}_{h}\right)_{r}=0, \quad \forall \boldsymbol{v}_{h} \in \boldsymbol{W}_{h, \triangleright}
$$

Now let $\boldsymbol{v}_{h}=\Pi_{h}^{c} \boldsymbol{S} \boldsymbol{w}_{h}-\boldsymbol{w}_{h}$. Then $\operatorname{curl}_{r z} \boldsymbol{v}_{h}=\Pi_{h}^{o} \operatorname{curl}_{r z}\left(\boldsymbol{S} \boldsymbol{w}_{h}\right)-\operatorname{curl}_{r z} \boldsymbol{w}_{h}$ by Theorem 5.1. Moreover, by $(6.6), \operatorname{curl}_{r z}\left(\boldsymbol{S} \boldsymbol{w}_{h}\right)=\operatorname{curl}_{r z} \boldsymbol{w}_{h}$. Since $\Pi_{h}^{o}$ is a projector, this implies that $\operatorname{curl}_{r z} \boldsymbol{v}_{h}=0$. Thus (6.7) implies

$$
\left\|\boldsymbol{S} \boldsymbol{w}_{h}-\boldsymbol{w}_{h}\right\|_{r} \leqslant\left\|\boldsymbol{S} \boldsymbol{w}_{h}-\Pi_{h}^{c} \boldsymbol{S} \boldsymbol{w}_{h}\right\|_{r} \leqslant C h^{\frac{1}{2}}\left\|\boldsymbol{S} \boldsymbol{w}_{h}\right\|_{H_{r}^{\frac{1}{2}}(D)} .
$$

Now, by a minor modification of [11, Theorem 3.2] to domains with Lipschitz boundary (using [13]) we obtain $\left\|\boldsymbol{S} \boldsymbol{w}_{h}\right\|_{H_{r}^{\frac{1}{2}(D)}} \leqslant C\left\|\operatorname{curl}_{r z} \boldsymbol{w}_{h}\right\|_{r}$. This completes the proof.

Proof of Theorem 6.1. If the result holds, then the wellposedness of problem (6.3) follows, so we only need to prove the error estimate for small enough $h$. Let $\boldsymbol{e}=\boldsymbol{u}-\boldsymbol{u}_{h}$, and let $\boldsymbol{w}_{h} \in \boldsymbol{W}_{h, \triangleright}$ be arbitrary. Then $A\left(\boldsymbol{e}, \boldsymbol{w}_{h}\right)=0$, so

$$
\begin{aligned}
\|\boldsymbol{e}\|_{\Lambda}^{2} & =\Lambda\left(\boldsymbol{e}, \boldsymbol{u}-\boldsymbol{w}_{h}\right)+\Lambda\left(\boldsymbol{e}, \boldsymbol{w}_{h}-\boldsymbol{u}_{h}\right) \\
& \leqslant\|\boldsymbol{e}\|_{\Lambda}\left\|\boldsymbol{u}-\boldsymbol{w}_{h}\right\|_{\Lambda}+A\left(\boldsymbol{e}, \boldsymbol{w}_{h}-\boldsymbol{u}_{h}\right)+\left(1+\kappa^{2}\right)\left(\boldsymbol{e}, \boldsymbol{w}_{h}-\boldsymbol{u}_{h}\right)_{r}, \\
& =\|\boldsymbol{e}\|_{\Lambda}\left\|\boldsymbol{u}-\boldsymbol{w}_{h}\right\|_{\Lambda}+\left(1+\kappa^{2}\right)\left(\boldsymbol{e}, \boldsymbol{w}_{h}-\boldsymbol{u}_{h}\right)_{r} .
\end{aligned}
$$

Next, we approximate $\left(\boldsymbol{e}, \boldsymbol{w}_{h}-\boldsymbol{u}_{h}\right)_{r}$ in two parts. Let $\boldsymbol{e}=\operatorname{grad}_{r z} \psi+\boldsymbol{\beta}$ be the unique Helmholtz decomposition in the weighted spaces, i.e., $\psi \in H_{r, \triangleright}^{1}(D)$ and $\boldsymbol{\beta} \in \boldsymbol{H}_{r, \triangleright}(\operatorname{curl}, D)$. A discrete Helmholtz decomposition in weighted spaces is also available from [11], which we use to decompose $\boldsymbol{w}_{h}-\boldsymbol{u}_{h}=\operatorname{grad}_{r z} \xi_{h}+\operatorname{curl}_{r z}^{\prime} s_{h}$ with $\xi_{h} \in V_{h, \triangleright}$ and $s_{h} \in S_{h}$. Then $\left(\operatorname{grad}_{r z} \psi, \operatorname{grad}_{r z} \xi_{h}\right)_{r}=\left(\boldsymbol{e}, \operatorname{grad}_{r z} \xi_{h}\right)_{r}=-\kappa^{-2} A\left(\boldsymbol{e}, \operatorname{grad}_{r z} \xi_{h}\right)=0$. Hence,

$$
\begin{aligned}
\left(\operatorname{grad}_{r z} \psi, \boldsymbol{w}_{h}-\boldsymbol{u}_{h}\right)_{r} & =\left(\operatorname{grad}_{r z} \psi, \operatorname{curl}_{r z}^{\prime} s_{h}\right)_{r}=\left(\operatorname{grad}_{r z} \psi, \operatorname{curl}_{r z}^{\prime} s_{h}-\boldsymbol{S}\left(\operatorname{curl}_{r z}^{\prime} s_{h}\right)\right)_{r} \\
& \leqslant\|\boldsymbol{e}\|_{r}\left\|\operatorname{curl}_{r z}^{\prime} s_{h}-\boldsymbol{S}\left(\operatorname{curl}_{r z}^{\prime} s_{h}\right)\right\|_{r} \\
& \leqslant C h^{\frac{1}{2}}\|\boldsymbol{e}\|_{r}\left\|\operatorname{curl}_{r z}\left(\operatorname{curl}_{r z}^{\prime} s_{h}\right)\right\|_{r}
\end{aligned}
$$

by Lemma 6.2. Thus, we have

$$
\left(\operatorname{grad}_{r z} \psi, \boldsymbol{w}_{h}-\boldsymbol{u}_{h}\right)_{r} \leqslant C h^{\frac{1}{2}}\|\boldsymbol{e}\|_{r}\left\|\operatorname{curl}_{r z}\left(\boldsymbol{w}_{h}-\boldsymbol{u}_{h}\right)\right\|_{r}
$$

which bounds a part of $\left(\boldsymbol{e}, \boldsymbol{w}_{h}-\boldsymbol{u}_{h}\right)_{r}$.

To bound the $\boldsymbol{\beta}$-component, let $\boldsymbol{z} \in \boldsymbol{H}_{r, \triangleright}(\operatorname{curl}, D)$ be the solution of

$$
A(\boldsymbol{z}, \boldsymbol{v})=(\boldsymbol{\beta}, \boldsymbol{v})_{r} \quad \forall \boldsymbol{v} \in \boldsymbol{H}_{r, \triangleright}(\operatorname{curl}, D) .
$$

Then,

$$
\|\boldsymbol{\beta}\|_{r}^{2}=(\boldsymbol{\beta}, \boldsymbol{e})_{r}=A(\boldsymbol{z}, \boldsymbol{e})=A\left(\boldsymbol{z}-\Pi_{h}^{c} \boldsymbol{z}, \boldsymbol{e}\right) \leqslant C\left\|\boldsymbol{z}-\Pi_{h}^{c} \boldsymbol{z}\right\|_{\Lambda}\|\boldsymbol{e}\|_{\Lambda} .
$$


By Theorem 5.1, $\left\|\boldsymbol{z}-\Pi_{h}^{c} \boldsymbol{z}\right\|_{r} \leqslant C h^{1 / 2}\|\boldsymbol{z}\|_{H_{r}^{\frac{1}{2}}(D)}$. Moreover, by the commutativity,

$$
\left\|\operatorname{curl}_{r z}\left(\boldsymbol{z}-\Pi_{h}^{c} \boldsymbol{z}\right)\right\|_{r}=\left\|\left(I-\Pi_{h}^{o}\right) \operatorname{curl}_{r z} \boldsymbol{z}\right\|_{r} \leqslant C h^{1 / 2}\left\|\operatorname{curl}_{r z} \boldsymbol{z}\right\|_{H_{r}^{\frac{1}{2}}(D)} \text {. }
$$

Hence,

$$
\begin{aligned}
& \|\boldsymbol{\beta}\|_{r}^{2} \leqslant C h^{\frac{1}{2}}\left(\|\boldsymbol{z}\|_{H_{r}^{\frac{1}{2}}(D)}+\left\|\operatorname{curl}_{r z} \boldsymbol{z}\right\|_{H_{r}^{\frac{1}{2}}(D)}\right)\|\boldsymbol{e}\|_{\Lambda} \\
& \leqslant C h^{\frac{1}{2}}\|\boldsymbol{\beta}\|_{r}\|\boldsymbol{e}\|_{\Lambda}
\end{aligned}
$$

by Lemma 6.1. This together with (6.9) implies that

$$
\left(\boldsymbol{e}, \boldsymbol{w}_{h}-\boldsymbol{u}_{h}\right)_{r} \leqslant C h^{\frac{1}{2}}\|\boldsymbol{e}\|_{\Lambda}\left\|\boldsymbol{w}_{h}-\boldsymbol{u}_{h}\right\|_{\Lambda} .
$$

Therefore, returning to (6.8),

$$
\begin{aligned}
\|\boldsymbol{e}\|_{\Lambda}^{2} & \leqslant\|\boldsymbol{e}\|_{\Lambda}\left\|\boldsymbol{u}-\boldsymbol{w}_{h}\right\|_{\Lambda}+C h^{\frac{1}{2}}\|\boldsymbol{e}\|_{\Lambda}\left\|\boldsymbol{w}_{h}-\boldsymbol{u}_{h}\right\|_{\Lambda} \\
& \leqslant\|\boldsymbol{e}\|_{\Lambda}\left\|\boldsymbol{u}-\boldsymbol{w}_{h}\right\|_{\Lambda}+C h^{\frac{1}{2}}\|\boldsymbol{e}\|_{\Lambda}\left\|\boldsymbol{w}_{h}-\boldsymbol{u}\right\|_{\Lambda}+C h^{\frac{1}{2}}\|\boldsymbol{e}\|_{\Lambda}^{2} .
\end{aligned}
$$

Thus, we have proved that for any $\boldsymbol{w}_{h} \in \boldsymbol{W}_{h, \triangleright}$,

$$
\left\|\boldsymbol{u}-\boldsymbol{u}_{h}\right\|_{\Lambda} \leqslant \frac{1+C h^{\frac{1}{2}}}{1-C h^{\frac{1}{2}}}\left\|\boldsymbol{u}-\boldsymbol{w}_{h}\right\|_{\Lambda},
$$

whenever $1-C h^{\frac{1}{2}}>0$, a condition satisfied for all $0<h<h_{0}$ if we choose $h_{0}<1 / C^{2}$.

\section{Appendix A. Proof of Propositions 2.1 And 3.1}

Both proofs involve scaling arguments where the weight function must be explicitly mapped. Due to the degeneracy of the weight function we must work with more than one reference domain, as we will see.

Proof of Proposition 2.1. If $K$ is an element that has no vertex on $\Gamma_{0}$, all the stated inequalities follow easily from their standard (unweighted) analogues, so we only need to prove that they hold also on the remaining $K \in \mathscr{T}_{h}$. These remaining elements can be classified in two types: For $n=1$ or 2, we say that a triangle $K$ is of type $n$ if $K$ has exactly $n$ vertices on $\Gamma_{0}$. We define two reference triangles in the $\hat{r} \hat{z}$-plane, namely, $\hat{K}_{1}=[(0,0),(1,1),(1,-1)]$ and $\hat{K}_{2}=[(0,-1),(1,0),(0,1)]$ (see e.g., [12, Figure 1]). Clearly, type $n$ triangles are in affine homeomorphism with $\hat{K}_{n}$.

We will prove only the last inequality as the others are similar. Let $K$ be of type 1 with vertices $\boldsymbol{a}_{i}$ such that $\boldsymbol{a}_{1}$ is on the $z$-axis. Let $F$ be the affine map that maps $\hat{K}_{2}$ one-one onto $K$ such that $\boldsymbol{a}_{1}$ is mapped to $(0,0)$. Let $v$ be mapped to $\hat{v}$ on $\hat{K}_{2}$ by $\hat{v}(\hat{r}, \hat{z})=v(r, z)$. Clearly, by the equivalence of norms on finite dimensional spaces, there exists a $\hat{C}$ depending only on $\ell$ such that

$$
\|\hat{v}\|_{L^{\infty}\left(\hat{K}_{2}\right)} \leqslant \hat{C}\|\hat{v}\|_{L_{\hat{r}}^{2}\left(\hat{K}_{2}\right)} .
$$


Setting $r_{i}=r\left(\boldsymbol{a}_{i}\right)$, let us note that $r=r_{2} \lambda_{2}+r_{3} \lambda_{3}$ is mapped under $F$ to $\hat{r}$. Hence, the right hand side can be bounded by

$$
\int_{\hat{K}_{2}} \hat{r}|\hat{v}|^{2} d \hat{\boldsymbol{x}}=\int_{K}\left(\lambda_{2}+\lambda_{3}\right)|v|^{2} \frac{\left|\hat{K}_{2}\right|}{|K|} d \boldsymbol{x} \leqslant \max \left(r_{2}^{-1}, r_{3}^{-1}\right) \int_{K} r|v|^{2} \frac{\left|\hat{K}_{2}\right|}{|K|} d \boldsymbol{x} .
$$

Since the $L^{\infty}$-norm is unchanged under the $F$-mapping, (A.1) implies

$$
r_{K} h_{K}^{2}\|v\|_{L^{\infty}(K)}^{2} \leqslant \hat{C}^{2} \max \left(\frac{r_{K}}{r_{2}}, \frac{r_{K}}{r_{3}}\right) \frac{h_{K}^{2}\left|\hat{K}_{2}\right|}{|K|}\|v\|_{L_{r}^{2}(K)}^{2} .
$$

By the shape regularity of $\mathscr{T}_{h}$, neither $r_{2}$ nor $r_{3}$ can be smaller than $C h$. Since $K$ has a vertex on the $z$-axis, we also know that $r_{K} \leqslant C h$. Hence (A.2) implies $r_{K} h_{K}^{2}\|v\|_{L^{\infty}(K)}^{2} \leqslant$ $C\|v\|_{L_{r}^{2}(K)}^{2}$ and the proof is complete for type 2 triangles.

For type 1 triangles, the proof uses similar arguments using a map $F$ that maps the edge of $\hat{K}_{1}$ on the $z$-axis to the edge of $K$ on the $z$-axis. We omit the details.

Proof of Proposition 3.1. We prove the result in each of the three cases of Figure 1. In each case, we have a different "reference" domain.

Case 1. In this case, $\boldsymbol{a}=\left(0, a_{z}\right)$ and $D_{\boldsymbol{a}}=\left\{(r, z): r^{2}+\left(z-a_{z}\right)^{2} \leqslant \rho^{2}\right.$ and $\left.r \geqslant 0\right\}$. The reference domain in this case is

$$
\hat{D}_{1}=\left\{(\hat{r}, \hat{z}): \hat{r}^{2}+\hat{z}^{2} \leqslant 1, \text { and } \hat{r} \geqslant 0\right\} .
$$

Consider the mapping

$$
\hat{r}=\frac{r}{\rho}, \quad \hat{z}=\frac{z-a_{z}}{\rho}, \quad\left(\text { or } \quad r=\hat{r} \rho, \quad z=\rho \hat{z}+a_{z}\right) .
$$

This map takes $\hat{D}_{1}$ one-one onto to $D_{\boldsymbol{a}}$ and the Jacobian is $\rho^{-2}$.

On the reference domain, define $\hat{\eta}_{1} \in \hat{P}_{\ell}$ by

$$
\int_{\hat{D}_{1}} \hat{r} \hat{\eta}_{1} \hat{p} d \hat{r} d \hat{z}=\hat{p}(\mathbf{0}) \quad \forall \hat{p} \in \hat{P}_{\ell},
$$

where $\hat{P}_{\ell}$ denotes the space of polynomials in $\hat{r}$ and $\hat{z}$ of degree at most $\ell$. Set

$$
\eta_{\boldsymbol{a}}(r, z)=\frac{1}{\rho^{3}} \hat{\eta}_{1}(\hat{r}, \hat{z})
$$

We will prove that this $\eta_{\boldsymbol{a}}$ satisfies all the stated properties.

To prove (3.1), we observe that by change of variables $\hat{p}(\hat{r}, \hat{z})=p(r, z)$,

$$
\begin{array}{rlrl}
\int_{D_{\boldsymbol{a}}} r \eta_{\boldsymbol{a}}(r, z) p(r, z) d r d z & =\int_{\hat{D}_{1}}(\hat{r} \rho) \frac{1}{\rho^{3}} \hat{\eta}_{1}(\hat{r}, \hat{z}) \hat{p}(\hat{r}, \hat{z}) \rho^{2} d \hat{r} d \hat{z} & & \text { by (A.4), } \\
& =\int_{\hat{D}_{1}} \hat{r} \hat{\eta}_{1} \hat{p} d \hat{r} d \hat{z}, & \\
& =\hat{p}(\mathbf{0})=p(\boldsymbol{a}), & \text { by (A.3), }
\end{array}
$$

for all $p \in P_{\ell}$. 
The estimate (3.2) follows from (3.1). Indeed, since $\eta_{\boldsymbol{a}} \in P_{\ell}$,

$$
\left\|\eta_{\boldsymbol{a}}\right\|_{r, D_{\boldsymbol{a}}}^{2}=\int_{D_{\boldsymbol{a}}} \eta_{\boldsymbol{a}}(r, z)^{2} r d r d z=\eta_{\boldsymbol{a}}(\boldsymbol{a})=\frac{\hat{\eta}_{1}(\mathbf{0})}{\rho^{3}} \leqslant \frac{C}{\rho^{2} r_{\boldsymbol{a}}} .
$$

Here we have used (A.4) and the fact that $\hat{\eta}_{1}$ is a fixed function on the reference domain and $r_{\boldsymbol{a}}=\rho$ in Case 1.

The last estimate (3.3) follows from (3.2) and Cauchy-Schwarz inequality:

$$
\left\|\eta_{\boldsymbol{a}}\right\|_{L_{r}^{1}\left(D_{\boldsymbol{a}}\right)}^{2} \leqslant\left(\int_{D_{\boldsymbol{a}}} r d \boldsymbol{x}\right)\left\|\eta_{\boldsymbol{a}}\right\|_{L_{r}^{2}\left(D_{\boldsymbol{a}}\right)}^{2} \leqslant \max _{\boldsymbol{x} \in D_{\boldsymbol{a}}} r(\boldsymbol{x})\left|D_{\boldsymbol{a}}\right| \frac{C}{\rho^{2} r_{\boldsymbol{a}}} \leqslant C \frac{\left(r_{\boldsymbol{a}}+\rho\right)}{r_{\boldsymbol{a}}}
$$

since $\left|D_{\boldsymbol{a}}\right|=\pi \rho^{2}$ and $r_{\boldsymbol{a}}=\rho$.

Case 2. Now $\boldsymbol{a}$ is a point in $\mathbb{R}_{+}^{2}$ not on the $z$-axis, and $D_{\boldsymbol{a}}=\left\{(r, z):\left(r-a_{r}\right)^{2}+\left(z-a_{z}\right)^{2} \leqslant\right.$ $\left.\rho^{2}\right\}$. The transformation

$$
\hat{r}=\frac{r-a_{r}}{\rho} \text { and } \hat{z}=\frac{z-a_{z}}{\rho}, \quad\left(\text { or } \quad r=a_{r}+\rho \hat{r}, \quad \text { and } \quad z=a_{z}+\rho \hat{z}\right)
$$

maps $D_{\boldsymbol{a}}$ one-one onto the "reference" domain $\hat{D}_{2}=\left\{(\hat{r}, \hat{z}): \hat{r}^{2}+\hat{z}^{2} \leqslant 1\right\}$. Define $\hat{\eta}_{2}(\hat{r}, \hat{z}) \in \hat{P}_{\ell}$ and $\hat{\eta}_{2, \alpha}(\hat{r}, \hat{z}) \in \hat{P}_{\ell}$ by

$$
\int_{\hat{D}_{2}} \alpha \hat{\eta}_{2, \alpha} \hat{p} d \hat{r} d \hat{z}=\hat{p}(\mathbf{0}) \quad \text { for all } \hat{p} \in \hat{P}_{\ell}
$$

for any positive function $\alpha(\hat{r}, \hat{z})$ bounded above and below on $\hat{D}_{2}$. Define $\eta_{\boldsymbol{a}} \in P_{\ell}$ by

$$
\eta_{\boldsymbol{a}}(r, z)=\frac{1}{\rho^{2}} \hat{\eta}_{2, \alpha}(\hat{r}, \hat{z}), \quad \text { with } \alpha=a_{r}+\rho \hat{r} .
$$

Note that when the linear function $r$ in the $r z$-plane is mapped over to the $\hat{r} \hat{z}$-plane by (A.6), we obtain the above $\alpha(\hat{r}, \hat{z})$. Hence, for any polynomial $\hat{p}(\hat{r}, \hat{z})=p(r, z)$,

$$
\begin{aligned}
\int_{D_{\boldsymbol{a}}} r \eta_{\boldsymbol{a}}(r, z) p(r, z) d r d z & =\int_{\hat{D}_{2}}\left(a_{r}+\rho \hat{r}\right) \frac{1}{\rho^{2}} \hat{\eta}_{2, \alpha}(\hat{r}, \hat{z}) \hat{p}(\hat{r}, \hat{z}) \rho^{2} d \hat{r} d \hat{z}=\int_{\hat{D}_{2}} \alpha \hat{\eta}_{2, \alpha} \hat{p} d \hat{r} d \hat{z} \\
& =\hat{p}(\mathbf{0})=p(\boldsymbol{a}),
\end{aligned}
$$

for all $p \in P_{\ell}$. This proves (3.1).

Next, to prove (3.2), we will first show that

$$
\hat{\eta}_{2, \alpha}(\mathbf{0}) \leqslant \frac{C}{\min _{\hat{\boldsymbol{y}} \in \hat{D}_{2}} \alpha(\hat{\boldsymbol{y}})} .
$$

Let $\hat{\eta}_{2}=\hat{\eta}_{2,1}$, i.e., $\hat{\eta}_{2}$ equals $\hat{\eta}_{2, \alpha}$ defined by (A.7) with $\alpha$ set to 1 . Then, since $\hat{\eta}_{2 \alpha} \in \hat{P}_{\ell}$,

$$
\begin{aligned}
\left\|\hat{\eta}_{2, \alpha}\right\|_{L_{\alpha}^{2}\left(\hat{D}_{2}\right)}^{2}=\hat{\eta}_{2, \alpha}(\mathbf{0}) & =\int_{\hat{D}_{2}} \hat{\eta}_{2} \hat{\eta}_{2, \alpha} d \hat{r} d \hat{z} \\
& \leqslant\left\|\hat{\eta}_{2}\right\|_{L^{2}\left(\hat{D}_{2}\right)}\left(\min _{\hat{\boldsymbol{y}} \in \hat{D}_{2}}(\alpha(\hat{\boldsymbol{y}}))\right)^{-1 / 2}\left\|\hat{\eta}_{2, \alpha}\right\|_{L_{\alpha}^{2}\left(\hat{D}_{2}\right)}
\end{aligned}
$$


Thus,

$$
\hat{\eta}_{2, \alpha}(\mathbf{0})=\left\|\hat{\eta}_{2, \alpha}\right\|_{L_{\alpha}^{2}\left(\hat{D}_{2}\right)}^{2} \leqslant \frac{C}{\min _{\hat{\boldsymbol{y}}_{\in} \in \hat{D}_{2}}(\alpha(\hat{\boldsymbol{y}}))}
$$

where $C=\left\|\hat{\eta}_{2}\right\|_{L^{2}\left(\hat{D}_{2}\right)}^{2}$ depends only on the fixed reference domain. This proves (A.9). Hence,

$$
\begin{aligned}
\left\|\eta_{\boldsymbol{a}}\right\|_{L_{r}^{2}\left(D_{\boldsymbol{a}}\right)}^{2} & =\eta_{\boldsymbol{a}}(\boldsymbol{a}) & & \text { by }(3.1), \text { since } \eta_{\boldsymbol{a}} \in P_{\ell}, \\
& =\frac{1}{\rho^{2}} \hat{\eta}_{2, \alpha}(\mathbf{0}) & & \text { by }(\mathrm{A} .8), \\
& \leqslant \frac{C}{\rho^{2} \min _{\hat{\boldsymbol{y}} \in \hat{D}_{2}}(\alpha(\hat{\boldsymbol{y}}))} & & \text { by }(\mathrm{A} .9), \\
& =\frac{C}{\rho^{2} \min _{\boldsymbol{y} \in D_{\boldsymbol{a}}}(r(\boldsymbol{y}))} . & &
\end{aligned}
$$

The last equality holds, since $\alpha(\hat{r}, \hat{z})=r$ at any point $(\hat{r}, \hat{z})$ mapped to $(r, z)$. This completes the proof of (3.2) for Case 2.

The proof of (3.3) proceeds as in Case 1 - see (A.5) - using (3.2).

Case 3. Now $D_{\boldsymbol{a}}$ is the closed disk with center $\tilde{\boldsymbol{a}}$ and radius $\rho$, where $\tilde{\boldsymbol{a}}=\left(\tilde{a}_{r}, \tilde{a}_{z}\right)$ is obtained by

$$
\left(\begin{array}{c}
\tilde{a}_{r} \\
\tilde{a}_{z}
\end{array}\right)=\left(\begin{array}{l}
a_{r} \\
a_{z}
\end{array}\right)+c\left(\begin{array}{rr}
\cos \theta & -\sin \theta \\
\sin \theta & \cos \theta
\end{array}\right)\left(\begin{array}{l}
\rho \\
0
\end{array}\right),
$$

for some fixed angle $\theta \geqslant 0$. Consider the mapping

$$
\left(\begin{array}{l}
\hat{r} \\
\hat{z}
\end{array}\right)=\frac{1}{\rho}\left(\begin{array}{rr}
\cos \theta & \sin \theta \\
-\sin \theta & \cos \theta
\end{array}\right)\left(\begin{array}{l}
r-a_{r} \\
z-a_{z}
\end{array}\right),
$$

or, equivalently,

$$
\left(\begin{array}{l}
r \\
z
\end{array}\right)=\left(\begin{array}{l}
a_{r} \\
a_{z}
\end{array}\right)+\rho\left(\begin{array}{rr}
\cos \theta & -\sin \theta \\
\sin \theta & \cos \theta
\end{array}\right)\left(\begin{array}{l}
\hat{r} \\
\hat{z}
\end{array}\right) .
$$

It is straightforward to show that this map sends the disk $D_{\boldsymbol{a}}$ in the $r z$-plane one-one onto the disk

$$
\hat{D}_{3}=\left\{(\hat{r}, \hat{z}):(\hat{r}-c)^{2}+\hat{z}^{2} \leqslant 1\right\}
$$

in the $\hat{r} \hat{z}$-plane. Since $c$ is fixed, $\hat{D}_{3}$ forms our third fixed "reference" domain. Note that the Jacobian of the change of variables from $(r, z)$ to $(\hat{r}, \hat{z})$ is again $\rho^{2}$.

As in the previous case, we now define $\hat{\eta}_{3, \alpha} \in \hat{P}_{\ell}$ by

$$
\int_{\hat{D}_{3}} \alpha \hat{\eta}_{3, \alpha} \hat{p} d \hat{r} d \hat{z}=\hat{p}(c, 0) \quad \text { for all } \hat{p} \in \hat{P}_{\ell},
$$

for any positive bounded function $\alpha(\hat{r}, \hat{z})$ on $\hat{D}_{3}$. Next, we define $\eta_{\boldsymbol{a}} \in P_{\ell}$ by

$$
\eta_{\boldsymbol{a}}(r, z)=\frac{1}{\rho^{2}} \hat{\eta}_{3, \alpha}(\hat{r}, \hat{z})
$$


after setting

$$
\alpha(\hat{r}, \hat{z})=a_{r}+\rho \hat{r} \cos \theta-\rho \hat{z} \sin \theta .
$$

It is obvious from (A.10) that this choice of $\alpha(\hat{r}, \hat{z})$ is obtained by mapping the linear function $r$ to the $\hat{r} \hat{z}$-plane. Therefore,

$$
\int_{D_{\boldsymbol{a}}} r \eta_{\boldsymbol{a}}(r, z) p(r, z) d r d z=\int_{\hat{D}_{3}} \alpha(\hat{r}, \hat{z}) \frac{1}{\rho^{2}} \hat{\eta}_{3, \alpha}(\hat{r}, \hat{z}) \hat{p}(\hat{r}, \hat{z}) \rho^{2} d \hat{r} d \hat{z}=\hat{p}(c, 0)=p(\boldsymbol{a}) .
$$

This proves (3.1).

The proofs of (3.2) and (3.3) proceed similarly as in the previous cases, after one proves that

$$
\hat{\eta}_{3, \alpha}(c, 0) \leqslant \frac{C}{\min _{\hat{\boldsymbol{y}} \in \hat{D}_{3}} \alpha(\hat{\boldsymbol{y}})},
$$

which is the analogue of (A.9) of Case 2.

\section{REFERENCES}

[1] D. N. Arnold, R. S. Falk, And R. Winther, Multigrid in $\boldsymbol{H}$ (div) and $\boldsymbol{H}$ (curl), Numer. Math., 85 (2000), pp. 197-217.

[2] — Finite element exterior calculus: from Hodge theory to numerical stability, Bull. Amer. Math. Soc. (N.S.), 47 (2010), pp. 281-353. DOI: 10.1090/S0273-0979-10-01278-4.

[3] F. Assous, P. Ciarlet, JR., And S. Labrunie, Theoretical tools to solve the axisymmetric Maxwell equations, Math. Methods Appl. Sci., 25 (2002), pp. 49-78.

[4] Z. Belhachmi, C. Bernardi, And S. Deparis, Weighted Clement operator and application to the finite element discretization of the axisymmetric Stokes problem, Numer. Math. (to appear), (2006).

[5] J. Bergh and J. Löfström, Interpolation Spaces. An Introduction, Springer-Verlag, New York, 1976.

[6] C. Bernardi, M. Dauge, and Y. Maday, Spectral methods for axisymmetric domains, vol. 3 of Series in Applied Mathematics (Paris), Gauthier-Villars, Éditions Scientifiques et Médicales Elsevier, Paris, 1999. Numerical algorithms and tests due to Mejdi Azaïez.

[7] F. Brezzi And M. Fortin, Mixed and Hybrid Finite Element Methods, no. 15 in Springer Series in Computational Mathematics, Springer-Verlag, New York, 1991.

[8] S. H. Christiansen and R. Winther, Smoothed projections in finite element exterior calculus, Math. Comp., 77 (2008), pp. 813-829.

[9] P. G. Ciarlet, Basic error estimates for elliptic problems, in Handbook of numerical analysis, Vol. II, P. G. Ciarlet and J.-L. Lions, eds., Handb. Numer. Anal., II, North-Holland, Amsterdam, 1991, pp. 17-351. Finite element methods. Part 1.

[10] PH. ClÉMEnT, Approximation by finite element functions using local regularization, RAIRO (Revue Française d'Atomatique, Informatique et Recherche Opérationnelle), Analyse Numérique, R-2 (9e année) (1975), pp. 77-84.

[11] D. Copeland, J. Gopalakrishnan, And M. OH, Multigrid in a weighted space arising from axisymmetric electromagnetics, Math. Comp, (2010).

[12] D. M. Copeland, J. Gopalakrishnan, and J. E. Pasciak, A mixed method for axisymmetric div-curl systems, Math. Comp., 77 (2008), pp. 1941-1965.

[13] M. Costabel, A remark on the regularity of solutions of Maxwell's equations on Lipschitz domains, Math. Methods Appl. Sci., 12 (1990), pp. 365-368.

[14] J. Gopalakrishnan and J. E. Pasciak, Overlapping Schwarz preconditioners for indefinite time harmonic Maxwell equations, Math. Comp., 72 (2003), pp. 1-15 (electronic). 
[15] — The convergence of $V$-cycle multigrid algorithms for axisymmetric Laplace and Maxwell equations, Math. Comp., 75 (2006), pp. 1697-1719.

[16] J. Gopalakrishnan, J. E. Pasciak, And L. F. Demkowicz, Analysis a multigrid algorithm for time harmonic Maxwell equations, SIAM J. Numer. Anal., 42 (2004), pp. 90-108 (electronic).

[17] P. Grisvard, Elliptic Problems in Nonsmooth Domains, no. 24 in Monographs and Studies in Mathematics, Pitman Advanced Publishing Program, Marshfield, Massachusetts, 1985.

[18] A. Kufner, Weighted Sobolev spaces, A Wiley-Interscience Publication, John Wiley \& Sons Inc., New York, 1985. Translated from the Czech.

[19] P. Monk, A finite element method for aproximating the time-harmonic Maxwell equations, Numer. Math., 63 (1992), pp. 243-261.

[20] P. Monk, Finite element methods for Maxwell's equations, Numerical Mathematics and Scientific Computation, Oxford University Press, New York, 2003.

[21] _ - A simple proof of convergence for an edge element discretization of Maxwell's equations, in Computational electromagnetics (Kiel, 2001), vol. 28 of Lect. Notes Comput. Sci. Eng., Springer, Berlin, 2003, pp. 127-141.

[22] J.-C. NÉdÉLEC, Mixed Finite Elements in $\mathbb{R}^{3}$, Numer. Math., 35 (1980), pp. 315-341.

[23] P.-A. Raviart and J. M. Thomas, A mixed finite element method for 2nd order elliptic problems, in Mathematical aspects of finite element methods (Proc. Conf., Consiglio Naz. delle Ricerche (C.N.R.), Rome, 1975), Springer, Berlin, 1977, pp. 292-315. Lecture Notes in Math., Vol. 606.

[24] A. H. Schatz, An observation concerning Ritz-Galerkin methods with indefinite bilinear forms, Math. Comp., 28 (1974), pp. 959-962.

[25] J. SchöBERL, Commuting quasi-interpolation operators for mixed finite elements, Tech. Rep. ISC01-10-MATH, Institute for Scientific Computation (ISC), Texas A\&M University, College Station, 2001.

[26] —_ A multilevel decomposition result in H(curl), in Proceedings of the 8th European Multigrid Conference, EMG 2005, P. Wesseling, C. Oosterlee, and P. Hemker, eds., TU Delft, 2008.

[27] — A posteriori error estimates for Maxwell equations, Math. Comp., 77 (2008), pp. 633-649.

University of Florida, Department of Mathematics, Gainesville, FL 32611-8105.

E-mail address: jayg@ufl.edu

James Madison University, Department of Mathematics and Statistics, Harrisonburg, VA 22807.

E-mail address: ohmx@jmu.edu 